\title{
Genome-wide and evolutionary analysis of the class III peroxidase gene family in wheat and Aegilops tauschii reveals that some members are involved in stress responses
}

\author{
Jun Yan ${ }^{1,2 \dagger}$, Peisen $\mathrm{Su}^{2+}$, Wen $\mathrm{Li}^{2}$, Guilian Xiao ${ }^{2}$, Yan Zhao ${ }^{2}$, Xin $\mathrm{Ma}^{2}$, Hongwei Wang ${ }^{2}$, Eviatar Nevo ${ }^{3^{*}}$ and
} Lingrang Kong ${ }^{2^{*}}$

\begin{abstract}
Background: The class III peroxidase (PRX) gene family is a plant-specific member of the PRX superfamily that is closely related to various physiological processes, such as cell wall loosening, lignification, and abiotic and biotic stress responses. However, its classification, evolutionary history and gene expression patterns are unclear in wheat and Aegilops tauschii.

Results: Here, we identified 374, 159 and 169 PRXs in Triticum aestivum, Triticum urartu and Ae. tauschii, respectively. Together with PRXs detected from eight other plants, they were classified into 18 subfamilies. Among subfamilies $V$ to XVIII, a conserved exon-intron structure within the "001" exon phases was detected in the PRX domain. Based on the analysis, we proposed a phylogenetic model to infer the evolutionary history of the exon-intron structures of PRX subfamilies. A comparative genomics analysis showed that subfamily VII could be the ancient subfamily that originated from green algae (Chlamydomonas reinhardtii). Further integrated analysis of chromosome locations and collinearity events of PRX genes suggested that both whole genome duplication (WGD) and tandem duplication (TD) events contributed to the expansion of T. aestivum PRXs (TaePRXs) during wheat evolution. To validate functions of these genes in the regulation of various physiological processes, the expression patterns of PRXs in different tissues and under various stresses were studied using public microarray datasets. The results suggested that there were distinct expression patterns among different tissues and PRXs could be involved in biotic and abiotic responses in wheat. QRT-PCR was performed on samples exposed to drought, phytohormone treatments and Fusarium graminearum infection to validate the microarray predictions. The predicted subcellular localizations of some TaePRXs were consistent with the confocal microscopy results. We predicted that some TaePRXs had hormone-responsive cis-elements in their promoter regions and validated these predicted cis-acting elements by sequencing promoters.
\end{abstract}

(Continued on next page)

\footnotetext{
* Correspondence: nevo@research.haifa.ac.il; Ikong@sdau.edu.cn

†Jun Yan and Peisen Su contributed equally to this work.

${ }^{3}$ Institute of Evolution, University of Haifa, 199 Aba Khoushy Ave., Mount Carmel, 3498838 Haifa, Israel

${ }^{2}$ State Key Laboratory of Crop Biology, Shandong Key Laboratory of Crop

Biology, College of Agronomy, Shandong Agricultural University, Tai'an,

Shandong 271018, People's Republic of China

Full list of author information is available at the end of the article
}

(c) The Author(s). 2019 Open Access This article is distributed under the terms of the Creative Commons Attribution 4.0 International License (http://creativecommons.org/licenses/by/4.0/), which permits unrestricted use, distribution, and reproduction in any medium, provided you give appropriate credit to the original author(s) and the source, provide a link to the Creative Commons license, and indicate if changes were made. The Creative Commons Public Domain Dedication waiver (http://creativecommons.org/publicdomain/zero/1.0/) applies to the data made available in this article, unless otherwise stated. 
(Continued from previous page)

Conclusion: In this study, identification, classification, evolution, and expression patterns of PRXs in wheat and relative plants were performed. Our results will provide information for further studies on the evolution and molecular mechanisms of wheat PRXs.

Keywords: Wheat class III peroxidase gene family, Conserved exon-intron structures, Collinearity events, Expression pattern

\section{Background}

Peroxidases (PRXs) are enzymes that catalyse the oxidation of many substrates by reducing hydrogen peroxide to water. They can be classified into two major groups: heme PRXs and non-heme PRXs. Heme PRXs can be further classified into two families: animal PRXs and non-animal PRXs. The non-animal PRXs contain three classes of PRXs, namely, class I, II and III PRXs [1]. Class I PRXs, such as microbial cytochrome c PRX, bacterial catalase-PRX and ascorbate PRX, are intracellular enzymes in plants, bacteria and yeast [2]. Class II PRXs are secreted oxidoreductive enzymes originating from fungi [3]. Class III PRXs are plant-specific secreted enzymes originating from plants [1].

Class III PRXs are involved in a broad range of physiological processes, such as cellular growth and cell wall loosening, lignification and suberisation, abiotic and biotic stress responses, fruit growth and ripening, and plant senescence [4]. By mediating the production of ROS (reactive oxygen species), a member of the cotton (Gossypium hirsutum) class III PRXs, GhPOX1, may play an important role during fibre cell elongation [5]. Arabidopsis thaliana class III PRX AtPRX17 is the direct target of the transcription factor AGL15 and regulates lignified tissue formation [6]. A. thaliana class III PRX AtPRX71 strengthens cell walls and restricts cell expansion in response to cell wall damage and during normal growth [7]. Four A. thaliana PRXs, AtPrx 4, 52, 49 and 72 , were predicted to be involved in lignification [8]. Using a knock-out mutant of AtPrx4, AtPrx4 was proven to be involved in cell wall lignification [9]. AtPrx52 was also proven to be involved in the synthesis of interfascicular fibres during the lignification process, and the suppression of AtPrx52 affected fibre lignification in Arabidopsis [10]. AtPrx72 is involved in lignin biosynthesis [11]. In proanthocyanidin-deficient $A$. thaliana seeds, class III PRXs are significantly activated relative to wild-type seeds, resulting in lower levels of $\mathrm{H}_{2} \mathrm{O}_{2}$ [12]. Overexpression of the class III PRXs AtPrx22, AtPrx39, and AtPrx69 increased cold tolerance in BRI1-GFP (green fluorescent protein) A. thaliana plants [13]. A putative Coffea arabica class III PRX was induced in response to root-knot nematode infection [14]. Solanum lycopersicum class III PRX LePrx17 was induced by JA (jasmonic acid) and pathogen infection, and LePrx09 was induced by ethephon, SA (salicylic acid), JA, pathogen infection, wounding and $\mathrm{H}_{2} \mathrm{O}_{2}$ stress [15]. Under arsenic (As) stress conditions, overexpression of the rice (Oryza sativa) class III PRX OsPRX38 in A. thaliana increased PRX, SOD (superoxide dismutase) and GST (glutathione-S-transferase) activity and enhanced lignification, resulting in reduced As accumulation [16]. Overexpression of $A$. thaliana class III PRX AtPrx64 in tobacco increased root growth and reduced the accumulation of aluminium (Al) and ROS in the roots, thereby improving tolerance to Al stress [17].

Only a few articles have reported the genome-wide identification of plant class III PRXs. In 2002, Tognolli et al. identified 73 class III PRXs in A. thaliana and analysed gene structures (intron/exon), gene duplication events, and expression patterns in different tissues (roots, stems, leaves and flowers) [18]. In 2004, Passardi et al. identified 138 rice class III PRXs and classified them into eight subfamilies (I-VIII) [19]. Despite the lack of complete plant genomes, they also studied the origin and expansion of class III PRXs by using EST (Expressed Sequence Tag) sequences and found 11-14 putative PRX sequences in Physcomitrella patens (moss) [19]. In 2014, Ren et al. identified 93 Populus trichocarpa class III PRXs and investigated the PtPRX expression patterns in five tissues (roots, shoots, leaves, buds, and phloem) and under abiotic stresses $\left(\mathrm{H}_{2} \mathrm{O}_{2}, \mathrm{SA}\right.$, salt, and drought) [20]. They found two large tandem-arrayed clusters of PtPRXs and identified seven positively selected sites in the four vacuole PtPRXs (PtPRX2, 3, 4, and 7). In 2015, Wang et al. identified 119 maize (Zea mays) class III PRXs and divided them into 18 groups [21]. They identified 16 related segmental duplication events and 12 tandem duplication events, calculated the $K a$ (nonsynonymous substitution) /Ks (synonymous substitution) values and found that most $Z m P R X s$ underwent purifying selection. Expression pattern analysis of $Z m P R X s$ was also performed using a public microarray dataset and qRT-PCR (quantitative real-time PCR) under $\mathrm{H}_{2} \mathrm{O}_{2}$, $\mathrm{SA}, \mathrm{NaCl}$ and PEG (polyethylene glycol) stress treatments. In 2016, Cao et al. identified 94 pear (Pyrus bretschneideri) class III PRXs and performed analyses of duplication events, conserved motifs, $\mathrm{Ka} / \mathrm{Ks}$ values and expression patterns by qRT-PCR [22].

Some articles studied the phylogeny of class III PRXs. In 2006, a class III plant PRX database, PeroxiBase, was published [23]. In 2015, a phylogenetic reconstruction of 
the non-animal PRX superfamily (class I-III PRXs, and others) was performed to trace their molecular evolution, and two additional class I members were identified [24]. In 2015, $\mathrm{Ka} / \mathrm{Ks}$ values of $62 \mathrm{~A}$. thaliana class III PRXs were calculated to examine their evolutionary divergence, and the nucleotide and amino acid substitutions of the duplicated genes AtPrx53-AtPrx54 and AtPrx36-AtPrx72 $(K a / K s>2)$ were identified as positive selection targets [25].

In this study, we performed genome-wide identification, evolution analysis and expression pattern analysis of class III PRXs in wheat and Aegilops tauschii. We identified PRXs in Triticum aestivum, Triticum urartu, Ae. tauschii, and eight other plant species and classified them into 18 subfamilies. We found that PRX subfamily VII first appeared in green algae (Chlamydomonas reinhardtii), and then VII and I appeared in moss ( $P$. patens). A conserved exon-intron structure within the "001" exon phases in the PRX domain was shared in subfamilies V-XVIII. An evolutionary model of PRX exon-intron structures was proposed. Chromosome locations and collinearity events of PRXs were identified in T. aestivum and related genomes to study the expansion and evolution of wheat PRXs. The expression patterns of tissues, abiotic stress responses and biotic stress responses were analysed using public microarray datasets. To validate the microarray predictions, qRT-PCR was performed on samples under drought conditions, samples with four phytohormone treatments and samples with Fusarium graminearum $(F g)$ infection. The confocal microscopy results validated the predicted subcellular localizations of some TaePRXs. Sequencing promoters of some TaePRXs validated the predicted hormone-responsive cis-elements. Our work will help researchers study the evolution and molecular mechanism of wheat PRXs.

\section{Results}

\section{Genome-wide identification and classification of class III} PRXs in wheat, Ae. tauschii and other plants

We searched the T. aestivum, T. urartu, and Ae. tauschii proteomes by using HMMER 3.1 and Pfam 30.0 in batch mode with the PRX domain (see Methods). The results showed that 374, 159 and 169 typical class III PRXs were identified in T. aestivum, T. urartu and Ae. tauschii, respectively. We also identified PRXs in eight other plant proteomes, including Brachypodium distachyon, $Z$. mays, O. sativa, A. thaliana, Vitis vinifera, Selaginella moellendorffii, $P$. patens and C. reinhardtii (Table 1, Additional file 13: Table S1). Atypical PRXs of these eleven plants, with less than 50\% PRX domain alignment, were excluded in the following analysis (Additional file 14: Table S2).
Table 1 The numbers of class III peroxidase gene families in 11 plants

\begin{tabular}{ll}
\hline Species & Number \\
\hline Chlamydomonas reinhardtii & 6 \\
Physcomitrella patens & 60 \\
Selaginella moellendorffii & 167 \\
Vitis vinifera & 85 \\
Arabidopsis thaliana & 81 \\
Zea mays & 156 \\
Oryza sativa & 125 \\
Brachypodium distachyon & 149 \\
Aegilops tauschii & 169 \\
Triticum urartu & 159 \\
Triticum aestivum & 374 \\
\hline
\end{tabular}

The classification of PRXs was based on two methods. First, the classification of PRXs in eleven plants was performed against the HMM (The hidden Markov model) based on the maize PRX alignments of Wang [21] (Additional file 13: Table S1). The results showed that PRXs were classified into I-XVIII subfamilies in T. aestivum, T. urartu and Ae. tauschii. Second, to verify the HMM classification, we constructed a neighbour-joining (NJ) phylogenetic tree of PRXs based on $T$. aestivum, $T$. urartu and Ae. tauschii truncated PRX domain sequences with the p-distance model and 1000 bootstraps (Fig. 1, Additional file 1: Figure S1a). A large NJ tree of PRXs was also constructed based on the truncated PRX domain sequences of these eleven plants (Additional file 1: Figure S1b). Interestingly, almost all PRXs belonging to the same clade indicated the same subfamily classification as that identified by HMMER. Large numbers of members were found in subfamilies I, V, VI and VII (Fig. 1). Some species-specific PRX clusters were found in $V$. vinifera, $S$. moellendorffii and P. patens. According to HMM and NJ classification, all six C. reinhardtii PRXs belong to subfamily VII, suggesting that PRX subfamily VII might be the ancient subfamily.

\section{Class III PRX evolution and conserved exon- intron structures}

We summarized the evolutionary process of plant class III PRXs by investigating the subfamily distributions in T. aestivum, $B$. distachyon, $S$. moellendorffii, $P$. patens and $C$. reinhardtii (Fig. 2a). The results indicated that subfamily VII first appeared in green algae (C. reinhardtii). Then, subfamilies VII and I were found in primitive plant moss (P. patens). The PRXs of fern (S. moellendorffii) expanded into six subfamilies, namely, VII, I, V, VI, IX and X. The PRXs of monocots (T. aestivum and $B$. distachyon) expanded into thirteen subfamilies, namely, VII, I, V, VI, IX, X, XII, XIV-XVIII and II. 


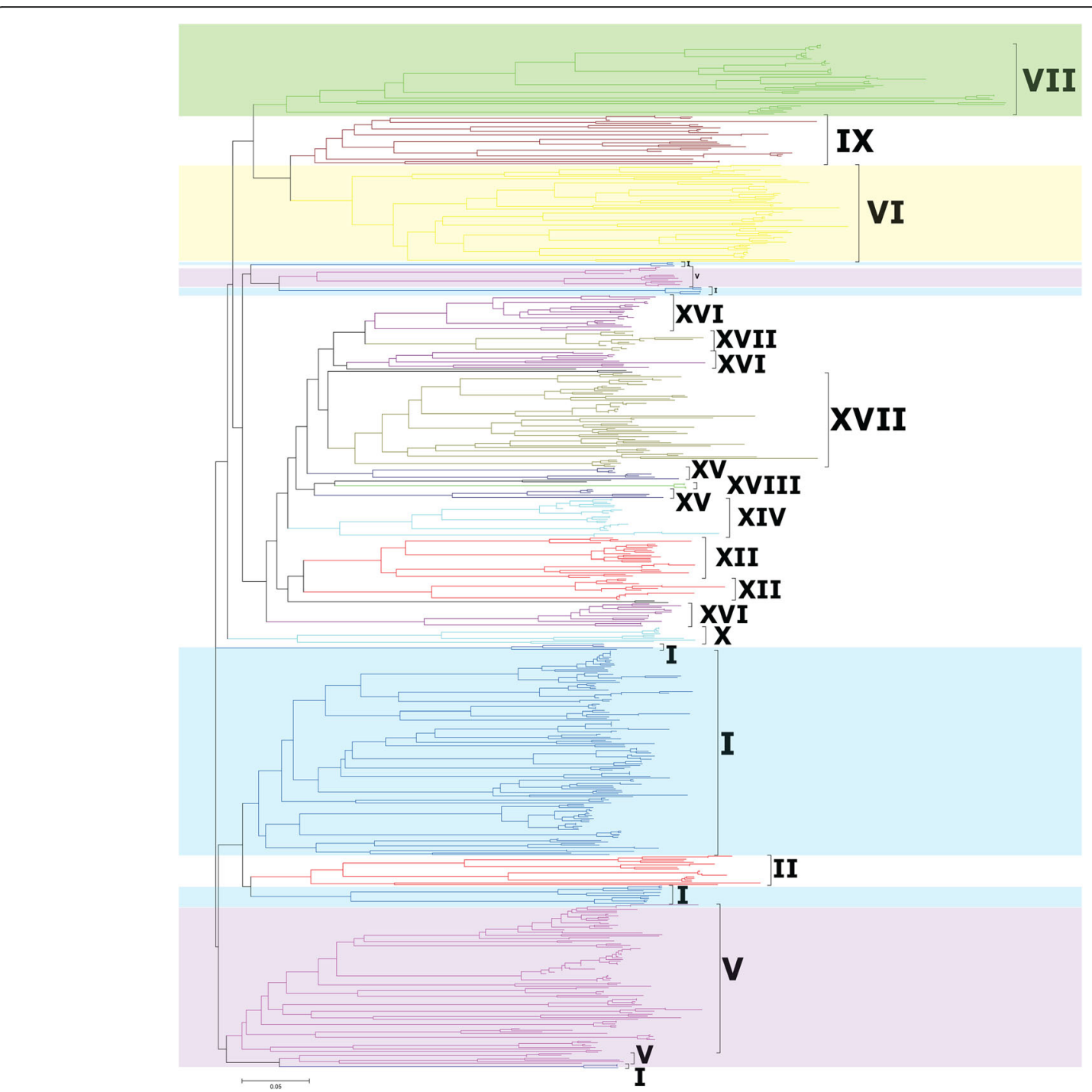

Fig. 1 Classification and phylogenetic relationships of the class III peroxidases in wheat and Ae. tauschii. The Neighbour-Joining tree was constructed by the amino acid sequences of the PRX domain using MEGA-CC 7.0 with the p-distance model. Major groups are labelled with different colours. Detailed information is provided in Additional file 1: Figure S1a

To obtain further insights into PRX evolution, we diagrammed the exon-intron structures within the PRX domain in the eleven investigated plants (Additional file 2: Figure S2). The results showed that some conserved exon-intron structures were present in the same subfamilies of PRXs across the investigated plants, especially in the PRX domain. We summarized these conserved exon-intron structures in $T$. aestivum, $B$. distachyon, $S$. moellendorffii, and P. patens (Additional file 3: Figure S3). For instance, the PRX subfamily I sequences of $T$. aestivum (Traes_1BS_8A9C9C25B.1), B. distachyon (BRADI2G37000.1), S. moellendorffii (EFJ22715), and P. patens (PP1S46_76V6.1) shared the same conserved exon-intron structure within the "01" exon phases in the PRX domain. Based on the above analysis, we noticed that most subfamilies V-XVIII shared the same conserved exon-intron structure within the "001" exon phases in the PRX domain (Fig. 2b, Additional file 3: Figure S3). These "001" exon phases could also be found in subfamily I but not in VII. Subfamily VII contained three types of conserved exon-intron structures, which were within the "1012-212", "2100-1020" and "0101-0000" exon phases in the PRX domain (Additional file 3: Figure S3).

\section{Chromosome locations and duplication events of $T$. aestivum class III PRXs}

We mapped T. aestivum class III PRXs to chromosome positions (Additional file 4: Figure S4, Additional file 15: Table S3). Two hundred and sixty-nine of $374 T$. 


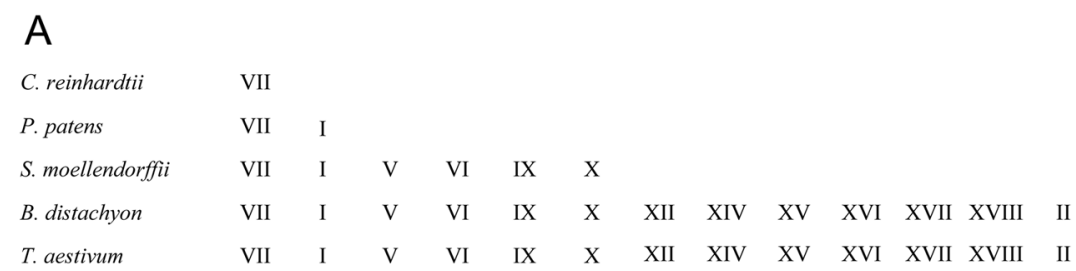

B

VII

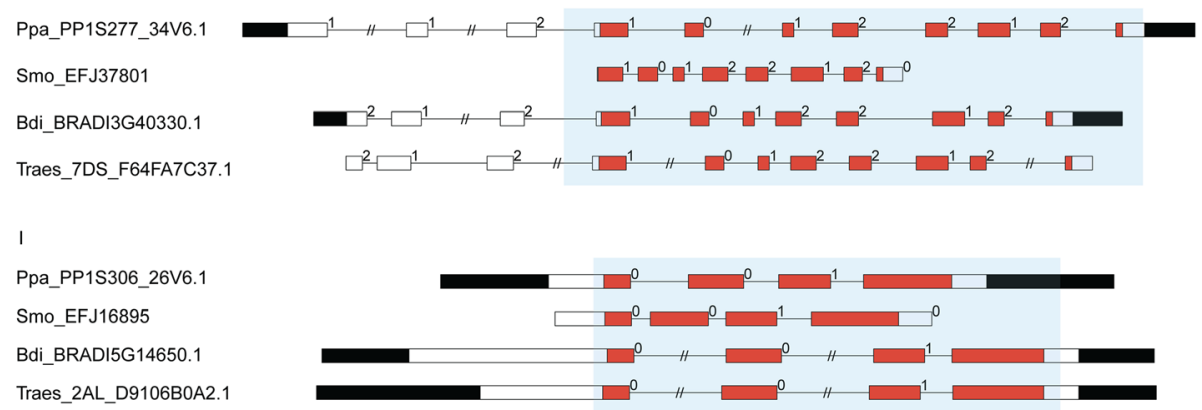

V-XVIII

Smo_EFJ33365

Bdi_BRADI3G33780.1

Traes_2BL_E83F2E30C.1

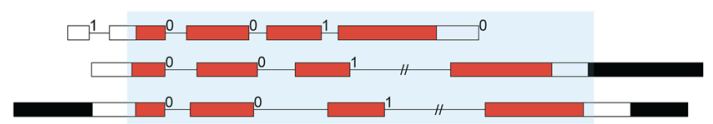

Fig. 2 Conserved exon-intron structures of PRXs. a The evolutionary history of the PRX subfamilies. $\mathbf{b}$ The diagram indicates that conserved exonintron structures with conserved exon phases were present in the PRX domain. Filled boxes: red represents the PRX domain; black boxes: untranslated regions (UTRs); white boxes: other exon regions; lines: introns; numbers 0, 1, and 2: exon phases. The lengths of the boxes and lines are scaled based on the lengths of the genes. The long introns are shortened by "//"

aestivum PRXs could be mapped on the chromosomes; the others were in scaffolds. These 269 T. aestivum PRXs were unevenly distributed among 21 chromosomes. Chromosomes 1A, 1B, 1D, 2A, 2B, 2D and 7D contained more PRXs than other T. aestivum chromosomes.

The allohexaploid bread wheat ( $T$. aestivum) genome consists of three subgenomes (A, B, and D), which are involved in three rounds of polyploidization [26]. To detect the relationship between PRX expansion and T. aestivum polyploidization, we identified 46 collinearity events by using MCscanX (Additional file 16: Table S4). The chromosome locations of related PRXs were distributed among chromosomes $1 \mathrm{~A}, 1 \mathrm{~B}, 1 \mathrm{D}, 2 \mathrm{~A}, 2 \mathrm{~B}, 2 \mathrm{D}, 4 \mathrm{~A}$, 4B, 4D, 5B, 5D, 6A, 6B, 6D and 7A (Fig. 3a). Our previous research demonstrated a peak $K s$ value of $0.03-0.45$ for collinearity events of T. aestivum PKs (protein kinase) [27]. In this study, we also found that most $K s$ values of T. aestivum PRXs were less than 0.45 , hinting that these T. aestivum PRX collinearity events could be attributed to $T$. aestivum polyploidization. Furthermore, we detected the PRX collinearity events among all synteny blocks in the T. aestivum genome (Fig. $3 \mathrm{~b}$ ).

To further infer the phylogenetic mechanism of the $T$. aestivum class III PRX gene family, comparative syntenic maps of T. aestivum associated with two Graminaceae (B. distachyon and O. sativa) were constructed (Fig. 3c-f, Additional file 16: Table S4). The results showed that 73 syntenic PRX gene pairs were detected between T. aestivum and B. distachyon (Fig. 3c). Similarly, 63 syntenic PRX gene pairs were detected between $T$. aestivum and O. sativa (Fig. 3e). We noticed that some syntenic PRX gene pairs shared the same $T$. aestivum PRX member associated with $B$. distachyon and $O$. sativa, suggesting that they might originate from a common ancestor before the Graminaceae split. For instance, the Bdi-Tae gene pair (BRADI1G20010.1 and Traes_2AS_ 5DF52D5D1.1) and Osa-Tae gene pair (Traes_2AS_ 5DF52D5D1.1 and OS07T0638600-00) shared the same T. aestivum PRX member (Traes_2AS_5DF52D5D1.1). We also calculated the $K a / K s$ values of syntenic PRX gene pairs between the T. aestivum A subgenome and T. urartu (86 gene pairs) and the T. aestivum D subgenome and Ae. tauschii (104) (Additional file 17: Table S5).

We identified 56 tandem $T$. aestivum PRX genes on 10 chromosomes, including $1 \mathrm{~B}, 1 \mathrm{D}, 2 \mathrm{~A}, 2 \mathrm{~B}, 3 \mathrm{~B}, 4 \mathrm{~A}, 4 \mathrm{D}$, 6A, 6D and 7D (Fig. 4, Additional file 18: Table S6). The genes formed 18 clusters on these 10 chromosomes. The number of members in each cluster ranged from 2 to 5 , 


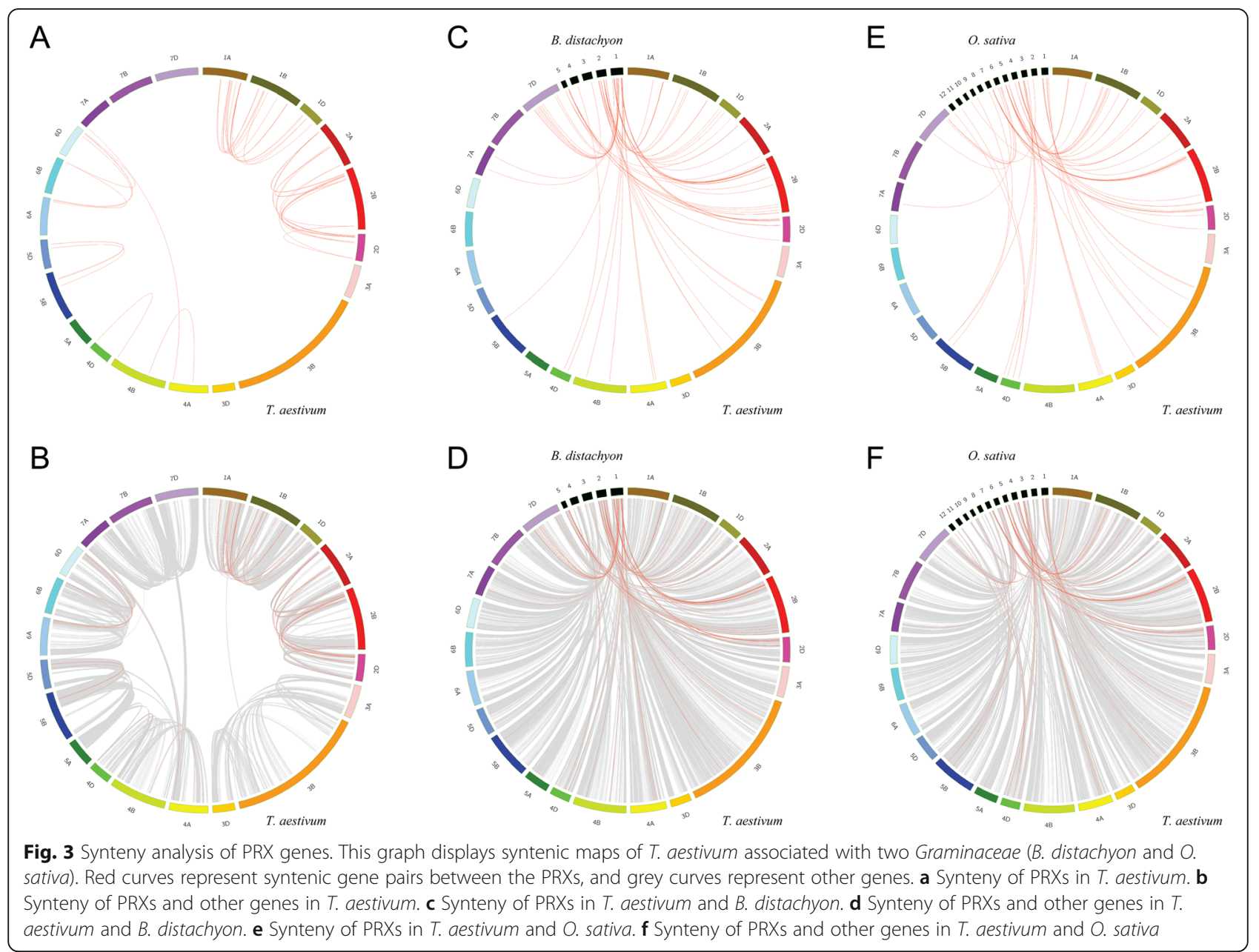

whereas the largest cluster was subfamily XVI, located on chromosome 7D.

\section{Expression patterns of $T$. aestivum class III PRXs in different tissues}

We performed a microarray-based expression pattern analysis of T. aestivum class III PRXs using 11 public datasets from the Affymetrix microarray GPL3802 platform. The results showed that 170 of 374 T. aestivum PRXs have probes in GPL3802. Based on the quality control of the NUSE (Normalized unscaled standard errors) and RLE (Relative log expression) diagrams (Additional file 5: Figure S5), we excluded 4 CEL files in the following analysis (Additional file 19: Table S7). The expression patterns of the 170 T. aestivum PRXs were investigated in different tissues, including three tissues (coleoptile, root and embryo) in the germinating seed stage, three tissues (root, crown and leaf) in the seedling stage, immature inflorescence tissue, three tissues (floral bracts, pistil and anthers) before anthesis, caryopsis tissue at 3-5 DAP (day after planting), embryonic tissue at 22 DAP, and endosperm tissue at 22 DAP (Additional file 6: Figure S6, Additional file 20: Table S8).
The results showed that some $T$. aestivum PRXs, such as Traes_2BL_2B45081D4.1 (VI), Traes_2AL_2224DCC1A.1 (VI) and Traes_4DL_8CE055F15.1 (VII), showed high expression levels in all investigated tissues. Similarly, some $T$. aestivum PRXs, such as Traes_4AL_0C8DFDE2B.1 (VI) and Traes_2AS_AB001AAB7.1 (XVII), exhibited low expression levels in all investigated tissues. We also noticed that some T. aestivum PRXs exhibited different expression levels in different tissues. For example, Traes_7DL_651CAFC08.1 (XVI) exhibited high expression level in the root of the seedling stage but relatively low expression levels in the other 11 tissues. Approximately 13 T. aestivum PRXs, such as Traes 7DL_6233C6F03.1 (I) and Traes_1AL_91E56EC8C.1 (V), exhibited high expression levels in two tissues, the root in the germinating seed stage and the root in the seedling stage, suggesting that they might participate in the development of the $T$. aestivum root.

\section{Subcellular localization of T. aestivum class III PRXs}

We selected four T. aestivum class III PRXs from different subfamilies to investigate their subcellular localization. The prediction websites WoLF PSORT and TargetP were 


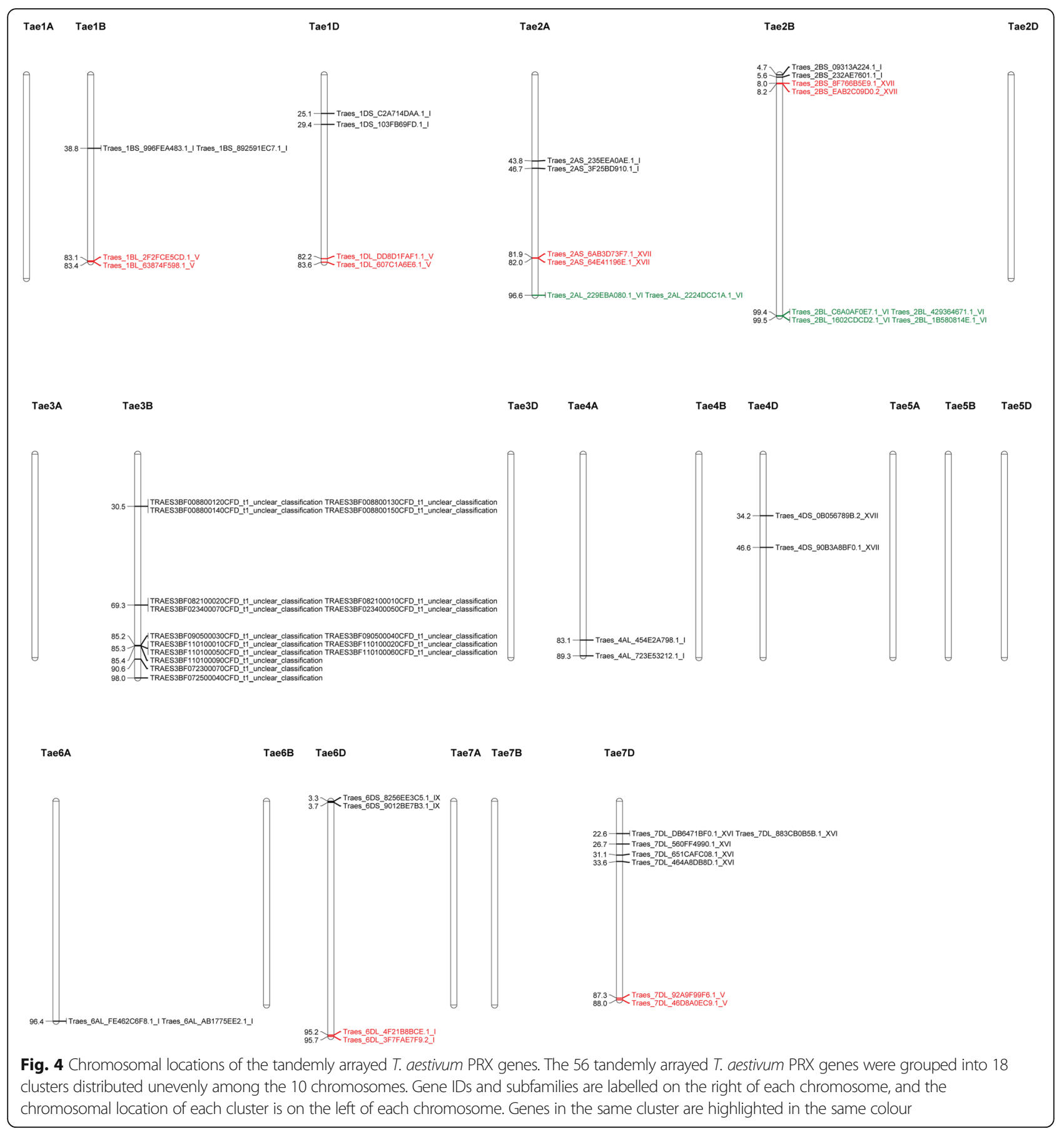

Table 2 Predicted and experimental subcellular localization of TaePRXs

\begin{tabular}{lllll}
\hline Gene ID & Subfamily & $\begin{array}{l}\text { Subcellular localization } \\
\text { (WoLF PSORT) }\end{array}$ & Subcellular localization (TargetP) & $\begin{array}{l}\text { Subcellular localization } \\
\text { (confocal microscopy) }\end{array}$ \\
\hline Traes_1AS_6C84785B3.2 & $\mathrm{I}$ & extr: 11, chlo: 1, mito: 1 & Secretory pathway & cell membrane \\
Traes_2AL_520618712.1 & $\mathrm{VI}$ & extr: 10, vacu: 4 & Secretory pathway & vacuole \\
Traes_6DL_2A99B8CDC.1 & $\mathrm{VII}$ & chlo: 13 & Chloroplast & thylakoid, chloroplast, cell membrane \\
Traes_6AS_5BAD56BB6.1 & $\mathrm{XVI}$ & chlo: 7, extr: 3, vacu: 2, nucl: 1 & Secretory pathway & vacuole, cell membrane, nucleus, cytoplasm \\
\hline
\end{tabular}


used to predict their subcellular localization (Table 2). To test the predicted subcellular localization of TaePRXs, Nterminal GFP-fused TaePRX proteins were expressed in tobacco leaves (Fig. 5). Interestingly, most of the predicted subcellular localizations were consistent with the confocal microscopy results (Table 2). For instance, Traes_6DL_ 2A99B8CDC.1 (VII) was predicted to be expressed in the chloroplast by WoLF PSORT and TargetP. Indeed, Traes_ 6DL_2A99B8CDC.1 (VII) was expressed in the chloroplast according to confocal microscopy.

\section{Expression patterns of $T$. aestivum class III PRXs under abiotic stress}

The expression patterns of $170 \mathrm{~T}$. aestivum class III PRXs were determined under various abiotic stress treatments (Additional file 7: Figure S7, Additional file 8: Figure S8, Additional file 21: Table S9). To search for the differentially expressed genes under abiotic stress, $T$. aestivum PRXs with $|\mathrm{FC}|>1.5$ (fold change) and $p<0.05$ were selected (Additional file 22: Table S10). (1) Cold: under cold treatment, most $T$. aestivum PRXs exhibited the same expression trend in the two wheat genotypes
(Freeze Resistance "SD16029" and Freeze Susceptible "SD16169"). For instance, Traes_2AL_2224DCC1A.1 (VI) and Traes_2BL_2B45081D4.1 (VI) showed upregulation in "SD16029" and "SD16169" under cold stress, hinting that these $T$. aestivum PRXs might regulate the cold stress response. Similarly, more PRXs, such as Traes 1DS_3D2F70A22.1 (I) and Traes_1AS_6C84785B3.2 (I), exhibited downregulation in "SD16029" and "SD16169" under cold stress. (2) Heat: under heat stress, most T. aestivum PRXs exhibited slight upregulation or downregulation. However, we also noticed that two PRXs, Traes 4DL_A6041E3DC.1 (V) and Traes_2AS_2B95E681C.1 (VII), exhibited dramatic upregulation under heat stress. (3) Drought: some PRXs exhibited slight upregulation or downregulation in both wheat genotypes (drought-susceptible "WL711" and drought-tolerant "C306") under drought stress. A few PRXs also showed reverse expression trends in "WL711" and "C306". For example, under drought stress, Traes_1DS_578ADDF80.1 (XII) exhibited upregulation in "WL711" but downregulation in "C306". (4) Nutrient deficiency: under the five nutrient-deficient stress treatments, most PRXs showed slight upregulation

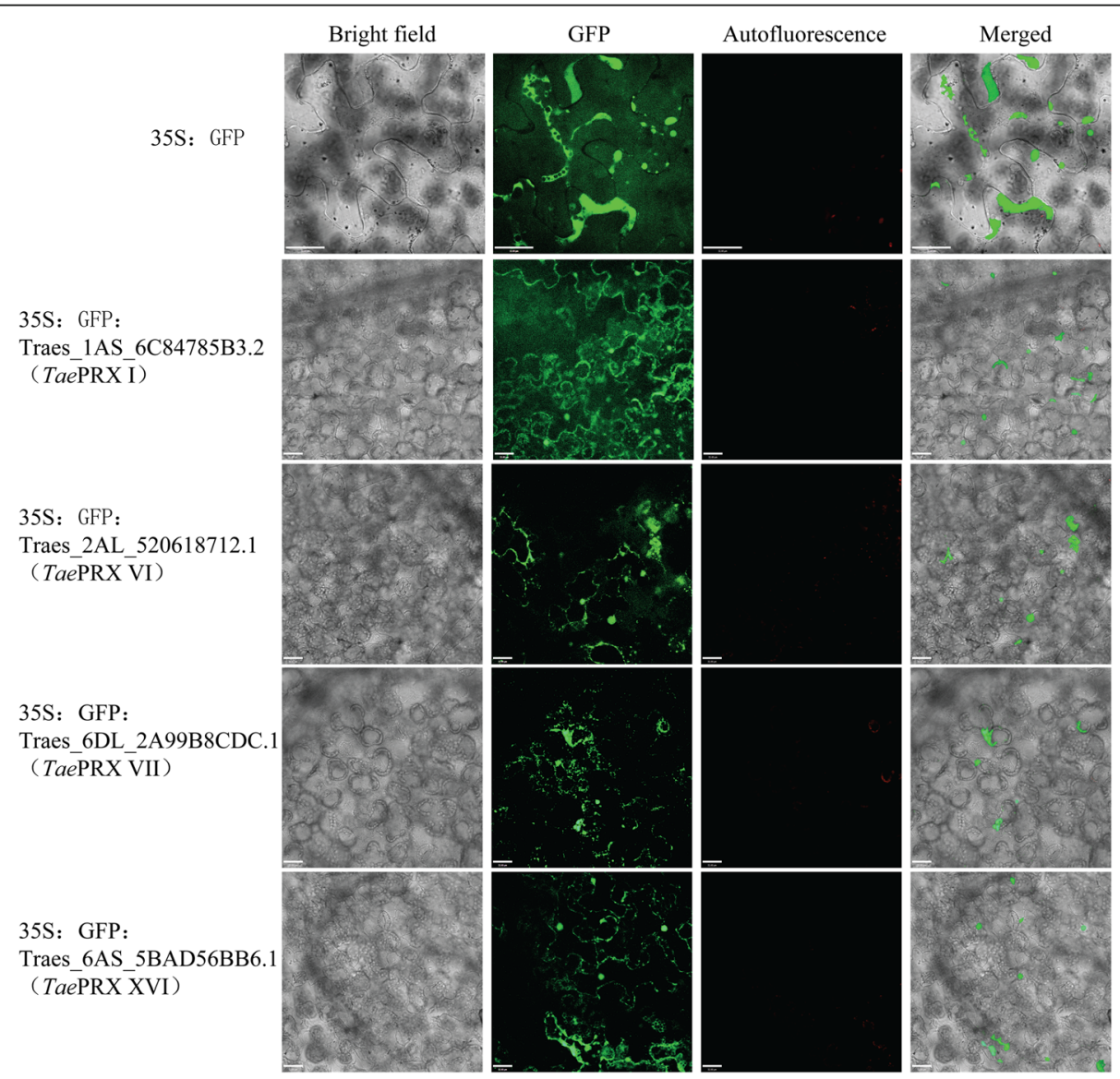

Fig. 5 The subcellular localization of TaePRX proteins in tobacco leaves. Localization of GFP signals from TaePRX proteins fused with GFP. Bright field, epifluorescence, chloroplast autofluorescence and merged images of tobacco leaves transfected with constructs expressing different fusion proteins. Bars $=32 \mu \mathrm{m}$ 
or downregulation. However, we also noticed that a few PRXs, such as Traes_2AS_3161D54F8.1 (XVII), Traes_ 7DL_D99ED7064.1 (XII) and Traes_7AL_80967149B.1 (XII), exhibited dramatic downregulation with only the no-phosphate fertilization treatment. Two PRXs, Traes_ 7DL_602B9D252.1 (XVI) and Traes_3AL_78711D4EB.1 (XII), showed dramatic upregulation with the no-sulphate fertilization treatment. (5) Seven phytohormones: most PRXs showed slight upregulation or downregulation under the seven phytohormones. For instance, Traes_3B_ 8732922B8.1 (VI) showed slight upregulation under all seven phytohormones (SA 0.19, MeJA (methyljasmonic acid) 0.08, GA (gibberellic acid) 0.33, ABA (abscisic acid) 0.48 , CK (trans-zeatin,one kind of cytokinins) 0.18, ET (ethylene) 0.55, and IAA (indole-3-acetic acid) 0.47). However, we also noticed that some $T$. aestivum PRXs, such as Traes_2AS 3161D54F8.1 (XVII), Traes_2DS 708F03DA3.1 (XVII) and Traes_2BS_B6EBC0962.1 (XVII), exhibited dramatic upregulation with only MeJA treatment. Similarly, some PRXs, such as Traes_2AL_2224DCC1A.1 (VI), Traes_5BL_3ED1B0234.2 (XVII) and Traes_1DL_
607C1A6E6.1 (V), exhibited dramatic downregulation with ABA treatment.

To further confirm the expression pattern analysis of the GEO (Gene Expression Omnibus) microarray, we selected eight $T$. aestivum PRXs to examine their expression in the cultivar "Sumai-3" under PEG (drought) treatment using qRT-PCR (Fig. 6). Some of the eight PRXs exhibited similar microarray and qRT-PCR results. (1) Traes_2DS_708F03DA3.1 (XVII): the $\log 2$ values of "WL711" (drought-susceptible) and "C306" (drought-tolerant) under drought conditions were 0.55 and 2.87 , while our qRT-PCR results showed upregulation at 6-72 h (h) after PEG treatment. (2) Traes_6AS_5BAD56BB6.1 (XVI): the microarray prediction of "C 306 " was consistent with our qRT-PCR results. The $\log 2$ value of "C306" was 2.05, while our qRT-PCR results exhibited upregulation at 6$72 \mathrm{~h}$. (3) Traes_2AS_AB001AAB7.1 (XVII): the $\log 2$ values of "WL711" and "C306" were 0.18 and 0.22 , respectively, while our qRT-PCR results showed upregulation at $6 \mathrm{~h}$. (4) Traes_2AL_A45F6AEBE.1 (VI): the log2 values of "WL711" and "C306" were 0.42 and 0.38 , respectively,

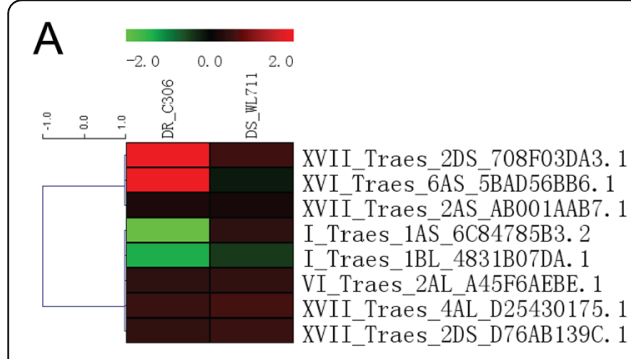

Traes_2AS_AB001AAB7.1

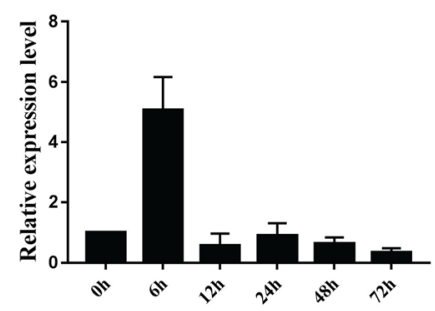

Traes_2AL_A45F6AEBE.1

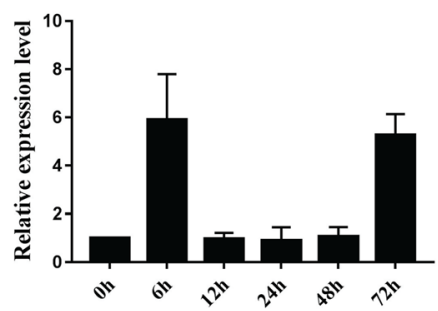

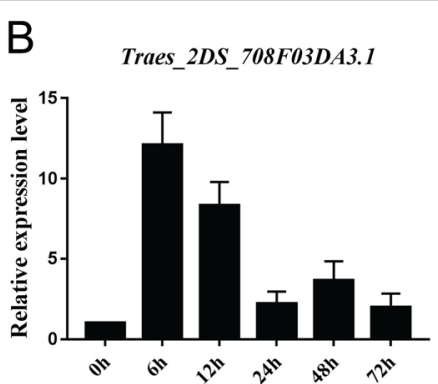

Traes_1AS_6C84785B3.2

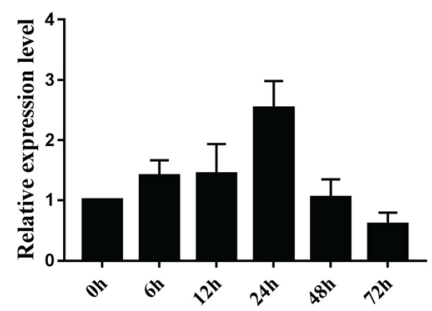

Traes_4AL_D25430175.1

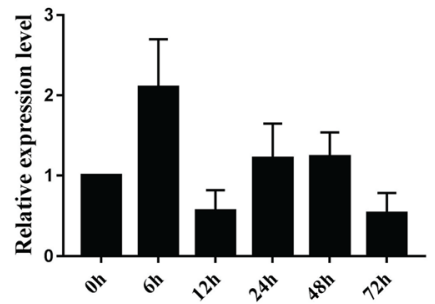

Traes_6AS_5BAD56BB6.1

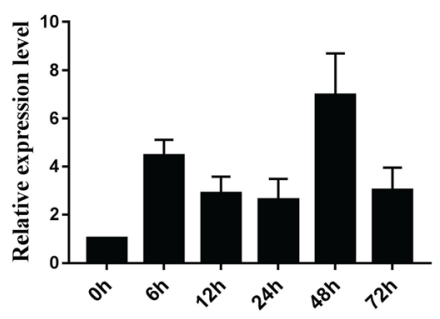

Traes_1BL_4831B07DA.1

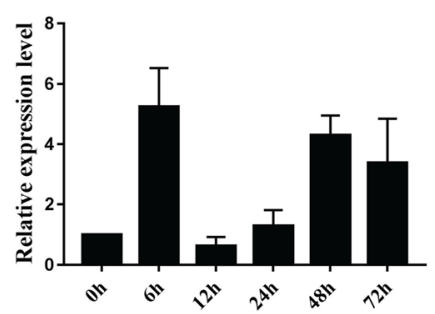

Traes_2DS_D76AB139C.1

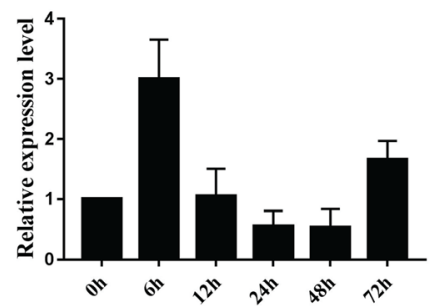

Fig. 6 Heatmap of eight selected T. aestivum PRXs and their qRT-PCR results under drought conditions. a Heatmap of the microarray. b qRT-PCR under PEG-600 treatment 
while our qRT-PCR results showed upregulation at 6 and 72 h. (5) Traes_4AL_D25430175.1 (XVII): the log2 values of "WL711" and "C306" were 0.59 and 0.51, respectively, while our qRT-PCR results showed upregulation at $6 \mathrm{~h}$. (6) Traes_2DS_D76AB139C.1 (XVII): the log2 values of "WL711" and "C306" were 0.52 and 0.44, respectively, while our qRT-PCR results showed upregulation at $6 \mathrm{~h}$.

To confirm the phytohormone microarray predictions, we also selected eight PRXs and examined their expression under four phytohormone (SA, JA, IAA and ABA) treatments using qRT-PCR (Fig. 7). Some of the eight PRXs exhibited similar microarray and qRT-PCR results. (1) Traes_4AL_D25430175.1 (XVII): the $\log 2$ values under SA, JA, IAA and ABA treatments were 0.14, 0.02, 0.11 and 0.02 , respectively, while our qRT-PCR results remained at the same expression level at 6 and $12 \mathrm{~h}$ (compared with $0 \mathrm{~h}$ ) under IAA and ABA treatments. Under JA treatment, this TaePRX exhibited upregulation at $1-48 \mathrm{~h}$ and reached peak expression at $24 \mathrm{~h}$. (2) Traes_2DS_D76AB139C.1 (XVII): the log2 values under SA, JA, IAA and ABA treatments were - 0.30, 0.80, 0.76 and 0.11 , respectively, while our qRT-PCR results showed dramatic upregulation at 1 and $6 \mathrm{~h}$ under IAA treatment. Under ABA treatment, this TaePRX exhibited slight upregulation at 1, 3, 6, 24 and $48 \mathrm{~h}$. With JA treatment, this TaePRX exhibited upregulation at 6-24 $\mathrm{h}$ and reached an approximately 20 -fold peak in expression at $24 \mathrm{~h}$. (3) Traes_2DS_2CCCA54C1.1 (XVII): the log2 values under SA, JA, IAA and ABA treatments were - 0.03 , $0.22,0.19$ and 0.20 , respectively, while our qRT-PCR results showed upregulation at 3-48 h under JA treatment. (4) Traes_5BL_3ED1B0234.2 (XVII): the log2 values under SA, JA, IAA and ABA treatments were $-0.12,0.44,-0.19$ and -2.57 , respectively, while our qRT-PCR results showed downregulation at $6 \mathrm{~h}$ under SA and IAA treatments. Under ABA treatment, this TaePRX exhibited downregulation at $12-48 \mathrm{~h}$. Under JA treatment, this TaePRX exhibited upregulation at $1 \mathrm{~h}$. (5) Traes_2BS_40C683B47.1 (XVII): the $\log 2$ values under SA, JA, IAA and ABA treatments were $-0.32,0.15,-0.28$ and -0.50 , respectively, while our qRT-PCR results showed upregulation at 3-24h under JA treatment. (6) Traes_6AS_5BAD56BB6.1 (XVI): the $\log 2$ values under SA, JA, IAA and ABA treatments were $-0.60,1.97,-0.81$ and -1.88 , respectively, while our qRT-PCR results showed downregulation at $1 \mathrm{~h}$ under SA and IAA treatments. Under JA treatment, this TaePRX exhibited upregulation at $1-24 \mathrm{~h}$. (7) Traes_2BS_ B6EBC0962.1 (XVII): the log2 values under SA, JA, IAA

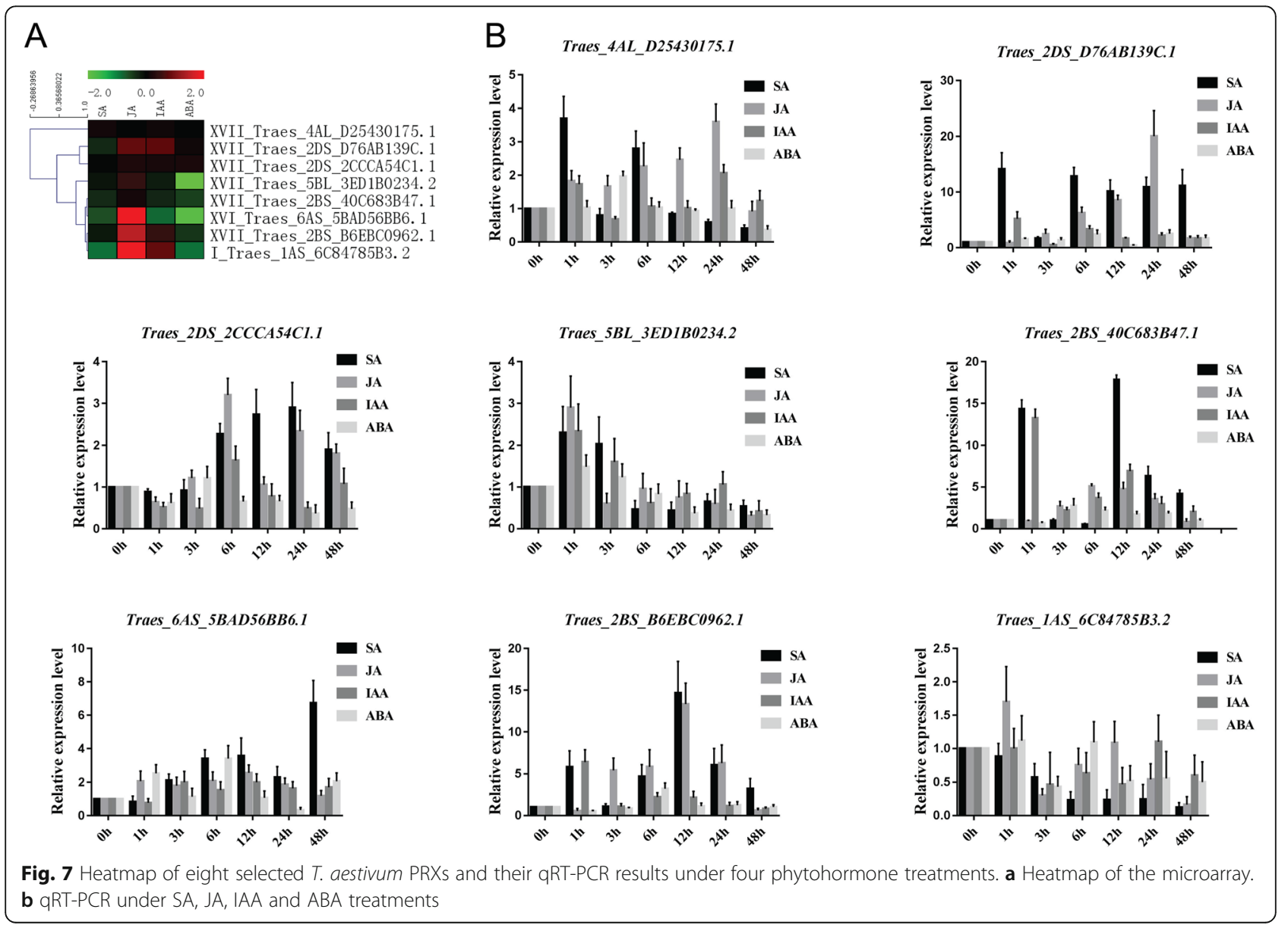


and $\mathrm{ABA}$ treatments were $-0.15,1.47,0.46$ and -0.42 , respectively, while our qRT-PCR results showed that this TaePRX reached an expression peak at $1 \mathrm{~h}$ and still exhibited slight upregulation at $6-12 \mathrm{~h}$ under IAA treatment. Under ABA treatment, this TaePRX exhibited slight downregulation at $1-3 \mathrm{~h}$. Under JA treatment, this TaePRX exhibited upregulation at $3-24 \mathrm{~h}$ and reached an approximately 12 -fold expression peak at $12 \mathrm{~h}$. (8) Traes 1AS_6C84785B3.2 (I): the $\log 2$ values under SA, JA, IAA and ABA treatments were $-0.87,2.72,0.82$ and -0.88 , respectively, while our qRT-PCR results showed that this TaePRX exhibited downregulation and slowly declined at 1-48 $\mathrm{h}$ under SA treatment. Under ABA treatment, this TaePRX exhibited downregulation at 3 and $12-48 \mathrm{~h}$. Under JA treatment, this TaePRX exhibited upregulation at $1 \mathrm{~h}$.

To study the mechanism of TaePRX expression induced by phytohormones, we predicted cis-acting elements in the upstream promoters of TaePRXs by using PlantCARE42 (Additional file 9: Figure S9 and Additional file 23: Table S11). The results showed that almost all investigated TaePRXs promoters contained the putative cis-acting elements responding to MeJA and ABA (Table 3). Some of these predicted results were consistent with the qRT-PCR and microarray results. For instance, the $\log 2$ value of Traes_2DS D76AB139C.1 (XVII) by microarray under ABA treatment was 0.11 , and the qRT-PCR results showed that this TaePRX exhibited slight upregulation at 1, 3, 6, 24 and $48 \mathrm{~h}$ under ABA treatment (Fig. 7). Indeed, the promoter of this TaePRX contained cis-acting elements responding to ABA.

To test these predicted cis-acting elements, sequencing validation of these upstream promoter sequences was performed (Additional file 10: Figure S10). The results showed that almost all predicted cis-acting elements were present in sequencing promoters. However, we found a cis-acting element within SNP (single nucleotide polymorphism) in Traes_6AS 5BAD56BB6.1 (XVI). This cis-acting element (GTGC AC) was responsive to ABA. SNP could also be found in other regions of the promoter of Traes_6AS 5BAD56BB6.1 (XVI).

We also compared four T. aestivum PRXs and their homologous maize PRXs [21] using qRT-PCR (Additional file 11: Figure S11). The results showed that three $T$. aestivum PRXs had similar expression trends as those of homologous maize PRXs under SA treatment. (1) Traes_4DS 90B3A8BF0.1 (XVII): both Traes_4DS_90B3A8BF0.1 and its homologous maize PRX (ZmPRX71 GRMZM2G171078_ T02) exhibited downregulation at $3 \mathrm{~h}$ under SA treatment. The expression of both genes recovered by one-fold at $6 \mathrm{~h}$. (2) Traes_2AS_E509AB03B.1 (XVII): the homologous maize PRX (ZmPRX26, GRMZM2G133475_T01) showed a 2.5fold upregulation at $12 \mathrm{~h}$ under SA treatment, while our qRT-PCR results showed that Traes_2AS_E509AB03B.1 exhibited an approximately 1.5 -fold upregulation at $12 \mathrm{~h}$ under SA treatment. (3) Traes_2AS_6AB3D73F7.1 (XVII): both Traes_2AS_6AB3D73F7.1 and its homologous maize PRX (ZmPRX75, GRMZM2G025441_T01) exhibited approximately 2-fold upregulation at $6 \mathrm{~h}$ under SA treatment.

\section{Expression patterns of T. aestivum class III PRXs under biotic stress}

We studied the expression patterns of $170 \mathrm{~T}$. aestivum class III PRXs under biotic stress treatments (Additional file 12: Figure S12, Additional file 24: Table S12). The differentially expressed genes of $T$. aestivum PRXs $(|\mathrm{FC}|>1.5$ and $p<0.05)$ under biotic stress were also determined (Additional file 25: Table S13). (1) Fusarium head blight (FHB): we noticed that a few T. aestivum PRXs, such as Traes_1BS_BA046E212.1 (XII), Traes 2BL_E8A65526C.1 (VI), Traes_7DL_602B9D252.1 (XVI) and Traes_2DS_D76AB139C.1 (XVII), exhibited progressive upregulation at 1-4 days (d) after $F g$ infection, suggesting that they might participate in the pathway responding to $F g$ infection. For instance, the $\log 2$ values of Traes_1BS_BA046E212.1 (XII) at 1, 2 and $4 \mathrm{~d}$ after $F g$ infection were $0.26,1.23$ and 2.71, respectively. (2) Powdery mildew: most $T$. aestivum PRXs exhibited the same expression trend but different $\log 2$ values with the two types of treatments. For example, the $\log 2$ values of

Table 3 Predicted and experimental cis-acting elements related to stress or hormone response

\begin{tabular}{llll}
\hline Gene ID & Subfamily & $\begin{array}{l}\text { Predicted cis-acting elements related } \\
\text { to stress or hormone response }\end{array}$ & $\begin{array}{l}\text { Experimental verification of cis-acting } \\
\text { elements using sequencing }\end{array}$ \\
\hline Traes_2DS_D76AB139C.1 & XVII & MeJA, GA, ABA, IAA & MeJA, GA, ABA, IAA \\
Traes_2DS_2CCCA54C1.1 & XVII & MeJA, SA, ABA, Low temperature, Drought inducibility & MeJA, ABA, Low temperature \\
Traes_5BL_3ED1B0234.2 & XVII & MeJA, SA, GA, ABA, Anaerobic induction, Drought inducibility & no sequencing \\
Traes_2BS_40C683B47.1 & $X V I I$ & MeJA, ABA, SA, Anaerobic_induction & MeJA, ABA, SA, Anaerobic_induction \\
Traes_6AS_5BAD56BB6.1 & XVI & SA, GA, ABA, Anaerobic induction, Drought inducibility & SA, GA, ABA, Anaerobic induction \\
Traes_2BS_B6EBC0962.1 & $X V I I$ & MeJA, IAA, Anaerobic induction, SA, ABA & MeJA, IAA, Anaerobic induction, SA \\
Traes_1AS_6C84785B3.2 & $\mathrm{I}$ & MeJA, ABA, Low temperature, Drought inducibility, SA & MeJA, ABA, Low temperature, \\
& & & Drought inducibility, SA \\
\hline
\end{tabular}


Traes_2AL_2224DCC1A.1 (VI) under $\mathrm{Si}(+$ ) (supply of soluble silicon) Bgt(+) (infection of Blumeria graminis $\mathrm{f}$. sp. tritici, $B g t)$ and $\mathrm{Si}(-) B g t(+)$ treatments were both upregulated at 1.53 and 0.57 , respectively. However, a few T. aestivum PRXs, such as Traes_4AL_0C8DFDE2B.1 (VI) and Traes_2AS_647C2FAA9.1 (I), exhibited opposite expression trends in the $\mathrm{Si}(+) B g t(+)$ and $\mathrm{Si}(-) B g t(+)$ treatments. (3) Blast disease: some T. aestivum PRXs, such as Traes_2BL_ B36482127.1 (VI), Traes_4AS_9EEABCE1C.1 (VII), Traes_ 4DL_8CE055F15.1 (VII) and Traes_1BL_4831B07DA.1 (I), were upregulated when infected with the three types of Magnaporthe pathogens. Similarly, three $T$. aestivum PRXs, Traes_6DL_2A99B8CDC.1 (VII), Traes_2AL_7EABAC855.1 (VII) and Traes_2AL_0A0101B75.1 (IX), showed downregulation under these three Magnaporthe pathogen infections. (4) Fly larval attack: we noticed that a few T. aestivum PRXs, such as TRAES3BF008800130CFD_t1 (unclear_classification), Traes_7DL_CE37E2AF1.1 (V), Traes_1DS A23800206.1 (II) and Traes_1AS_C70D49E2E.4 (VI), exhibited progressive upregulation at 6,12 and $24 \mathrm{~d}$ after fly larval attack. For instance, the $\log 2$ values of Traes_1DS A23800206.1 (II) at 6, 12 and $24 \mathrm{~d}$ were $0.84,0.97$ and 1.14, respectively. Similar to FHB, these PRXs might also regulate the pathway of the fly larval attack response. (5) Earthworm: most T. aestivum PRXs exhibited the same expression trend but different $\log 2$ values under both treatments. For instance, the $\log 2$ values of Traes_6DL_2A99B8CDC.1 (VII) were 0.04 and 0.77 in the earthworm $(+) G g t(+)$ (infection by the soilborne fungus Gaeumannomyces graminis var. tritici, Ggt) and earthworm $(+) \operatorname{Ggt}(-)$ treatments, respectively. We also noticed that some PRXs, such as Traes_7DS_F64FA7C37.1 (VII) and Traes_2AS_647C2FAA9.1 (I), exhibited opposite expression trends under earthworm(+) Ggt(+) and earthworm $(+) G g t(-)$ treatments.

To confirm the FHB expression pattern of the GEO microarray, we examined eight $T$. aestivum PRXs using qRT-PCR (Fig. 8). The log2 values of these eight T. aestivum PRXs were all upregulated at 1, 2 and $4 \mathrm{~d}$ after $F g$ infection. This result was consistent with our qRT-PCR results that all exhibited upregulation at $48-96 \mathrm{~h}$ after $\mathrm{Fg}$ infection. Interestingly, four T. aestivum PRXs, Traes_2AL_ A45F6AEBE.1 (VI), Traes_5DL_011018E3C.1 (IX), Traes 1BS_871E20CF0.1 (I) and Traes_2AS_AB001AAB7.1 (XVII), showed more than 100-fold upregulation at 48-96 $\mathrm{h}$ after $\mathrm{Fg}$ infection, hinting that they might be important for the signalling pathways of the $F g$ infection response.

\section{Discussion}

Evolution and duplication events of the class III PRX gene family in wheat and Ae. tauschii

In this study, we determined the identification, evolution and expression of the class III PRX gene family in wheat and Ae. tauschii. The numbers of PRX gene families in T. urartu (159) and Ae. tauschii (169) are similar to those in three investigated monocots (B. distachyon, 149, $O$. sativa, 125 , and $Z$. mays, 156) but higher than that in two eudicots ( $V$. vinifera, 85, and A. thaliana, 81). This is consistent with a report that maize PRXs (119) are higher in number than Arabidopsis (73) and poplar (93) [21]. The number of PRXs in T. aestivum (374) is approximately three-fold higher than that in $T$. urartu (159) and Ae. tauschii (169). This "three-fold" relationship is consistent with our previous article on the PK gene family in which the number of PKs in T. aestivum (3269) is approximately three-fold greater than that in $T$. urartu (1213) and Ae. tauschii (1448) [27]. This is because the origin of allohexaploid bread wheat (T. aesti$v u m$ ) was involved in three polyploidizations [26]. The examples of the "three-fold" relationship are presented in greater detail in Additional file 1: Figure S1. For instance, five sequences (Traes_7AS_81327ECA0.1, TRIUR3_11069-P1, Traes_7BS_3878BF050.1, EMT10887 and Traes_7DS_F0152FC36.1) formed a clan with a bootstrap value of 100 .

To infer the evolutionary history of the PRX gene family, we also identified PRXs in eight other plants, including primitive green algae $(6, C$. reinhardtii), moss $(60, P$. patens) and fern (167, $S$ moellendorffii). Our results showed that the PRX subfamily VII first appeared in C. reinhardtii. In 2004, Passardi et al. [19] found 11-14 putative PRXs in $P$. patens using the EST database but none in C. reinhardtii, Thalassiosira pseudonana or Phytophthora sojae. The absence of $C$. reinhardtii PRX in the Passardi report may be because of the incomplete $C$. reinhardtii genome at that time or due to use of the TBLASTN search method. The conserved exon-intron structure with exon phase "001" in the PRX domain could be found in most of the subfamilies V-XVIII, from S. moellendorffii to T. aestivum, hinting that this exonintron structure might be important for the function of PRXs. This "001" exon-intron structure could also be found in P. patens (PP1S306_26V6.1), but not in C. reinhardtii, suggesting that the ancestral sequence of subfamilies V-XVIII might have appeared in mossresembling ancestors. We did not found PRX subfamily II in two investigated eudicots ( $V$. vinifera and $A$. thaliana) but found it in all investigated monocots, and its "001" exon-intron structure had changed into a "0" twoexon structure, suggesting that a monocot-specific exon fusion event occurred in the PRX subfamily II after the monocot-eudicot split.

Based on the analysis of PRXs across C. reinhardtii to $T$. aestivum, we proposed a model to infer the evolution of the PRX gene family (Fig. 9). First, the PRX subfamily VII might have appeared in an algae-resembling ancestor, but their exon-intron structures were algae-specific. Indeed, we cannot find the four types of exon-intron structures of the C. reinhardtii subfamily VII PRXs in 


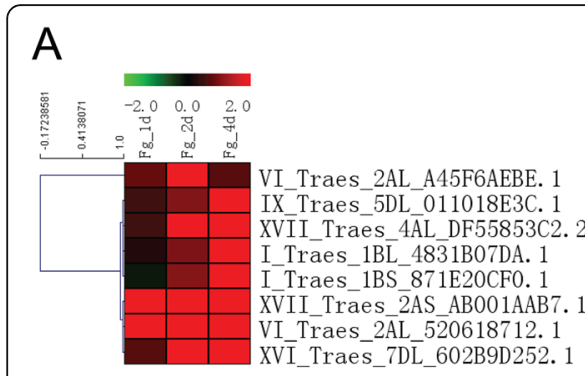

Traes_4AL_DF55853C2.2

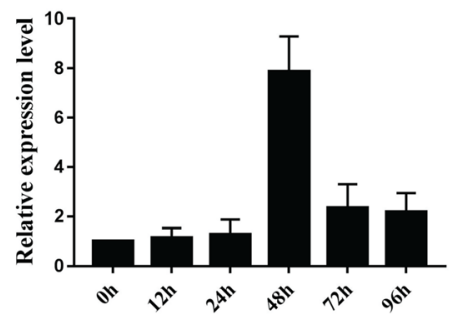

Traes_2AS_AB001AAB7.1

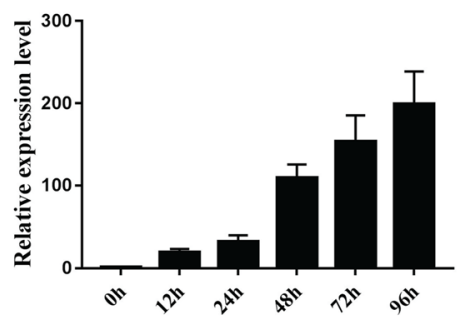

B

Traes_2AL_A45F6AEBE.1

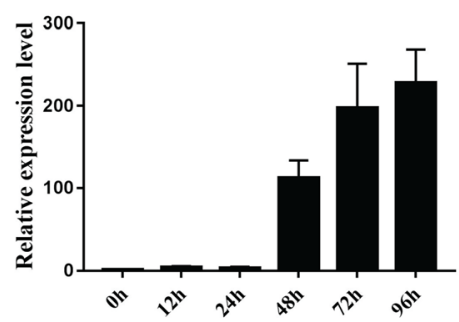

Traes_1BL_4831B07DA.1

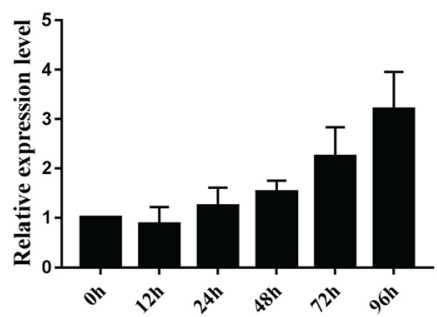

Traes_2AL_520618712.1

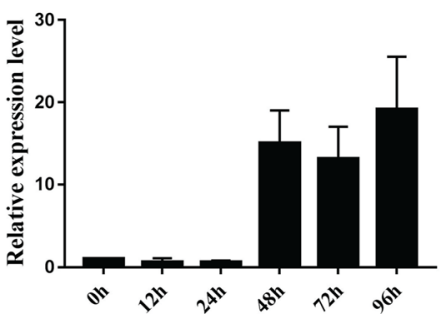

Traes_5DL_011018E3C.1

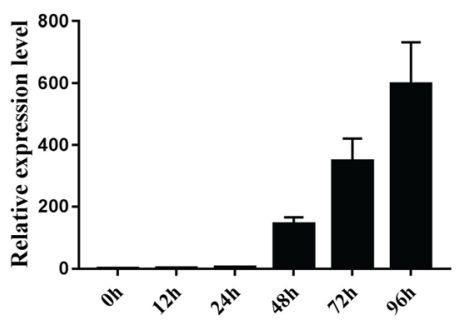

Traes_1BS_871E20CF0.1

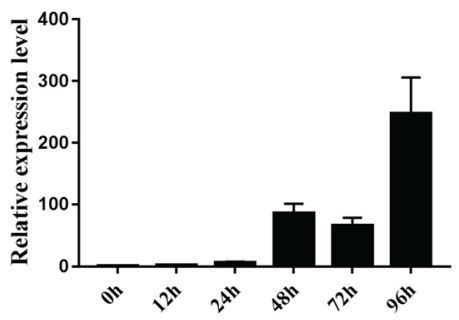

Traes_7DL_602B9D252.1

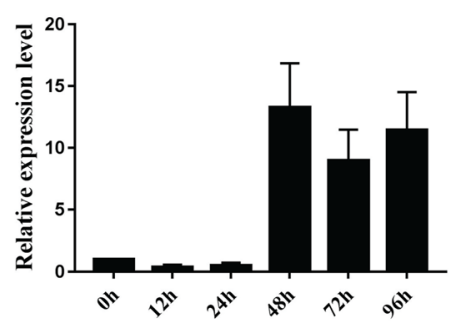

Fig. 8 Heatmap of eight selected T. aestivum PRXs and their qRT-PCR results with Fg infection. a Heatmap of the microarray. $\mathbf{b}$ qRT-PCR with Fg infection

other investigated plants. Second, PRX subfamilies VII and I might appear in a moss-resembling ancestor. Two types of conserved exon-intron structures ("1012-212" and "2100-1020") appeared in moss PRX subfamily VII, and they were conserved from $P$. patens to $T$. aestivum. The conserved exon-intron structure within exon phase "0101-0000" appeared in S. moellendorffii PRX subfamily VII, and it was conserved from $S$. moellendorffii to T. aestivum. In contrast to the abundant exon-intron structures of subfamily VII, PRX subfamily I contained four sample conserved exon-intron structures ("001", "01", "0" and "00"). Third, except for subfamilies VII and I, the new PRX subfamilies V, VI, IX and X appeared in S. moellendorffii, but their exon-intron structures were all "001". Therefore, we inferred that PRX subfamilies V, VI, IX and $\mathrm{X}$ might originate in subfamily $\mathrm{I}$. Interestingly, subfamilies XII, XIV-XVIII and II appeared in eudicots and monocots, but most of their exon-intron structures were still "001". In summary, most PRX subfamilies contained conserved exon-intron structure "001", which might originate in subfamily I.

It was reported that both segmental and tandem duplication contributed to the expansion of maize PRXs [21], while segmental duplication mainly contributed to the expansion of Chinese pear PRXs [22]. In this study, we determined $46 T$. aestivum PRX collinearity events contributed by segmental duplication (Additional file 16: Table S4), and there were 56 tandem T. aestivum PRXs (Additional file 18: Table S6), suggesting that both segmental and tandem duplication contributed to the expansion of T. aestivum PRXs. The number of orthologous genes (73) between $T$. aestivum and $B$. distachyon is more than that (63) between T. aestivum and rice (Additional file 16: Table S4), suggesting that the split between $T$. aestivum and $B$. distachyon progenitors occurred after rice diverged from the common ancestor of $T$. aestivum and $B$. distachyon. Indeed, it was reported that Triticeae and Brachypodieae are sister clades and that Oryza is located on the root of the cluster 


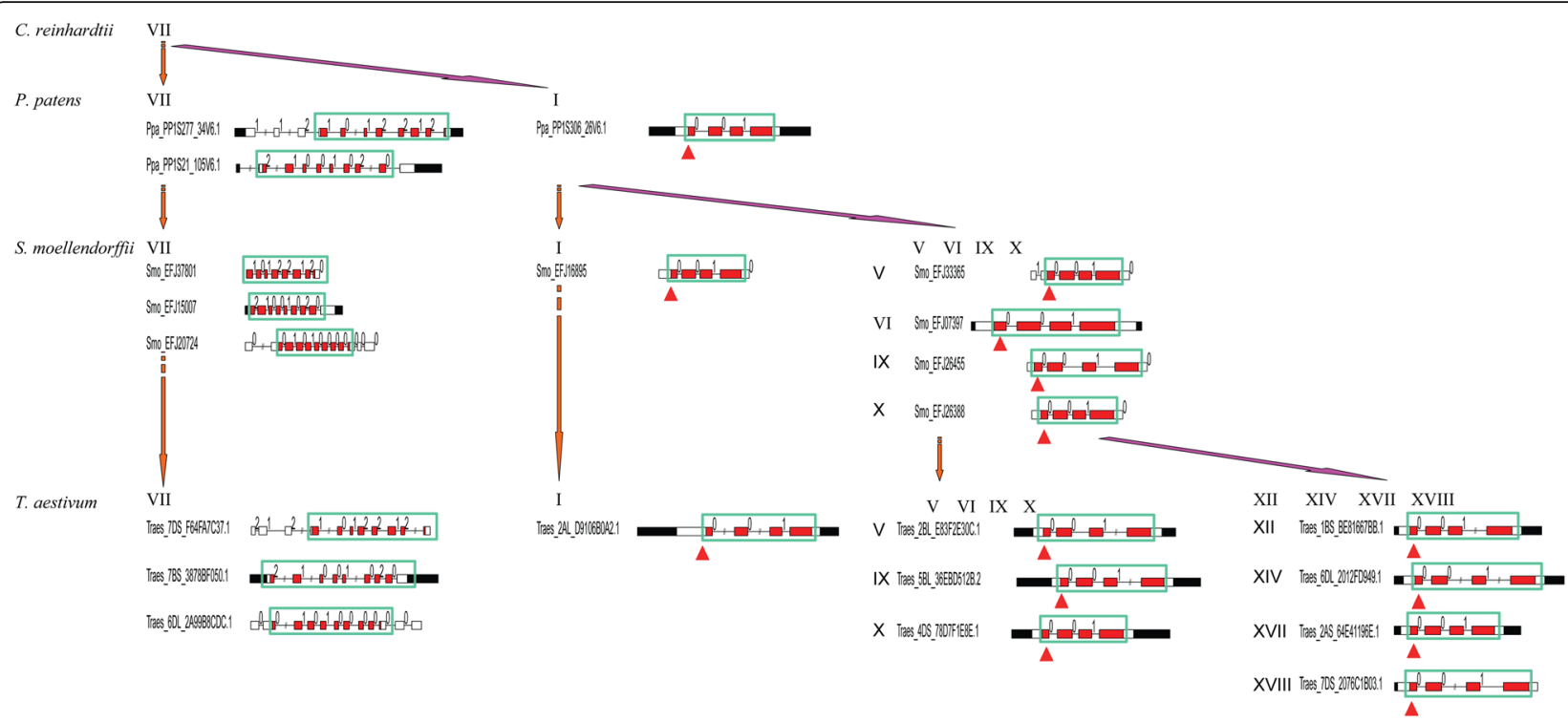

Fig. 9 The evolutionary model of the exon-intron structure of PRX genes. This graph displays the evolution of PRX subfamilies in four representative plants (C. reinhardtii, P. patens, S. moellendorffii and T. aestivum). We found that a conserved exon-intron structure with exon phase "001" in the PRX domain was present in many PRX subfamilies of the investigated plants. These "001" structures are circled by a green box and red arrow. The descriptions of the domain and exon phases are the same as those in Fig. 2

containing six sister clades (Diarrheneae, Brachypodieae, Poeae, Aveneae, Bromeae and Triticeae) [28].

\section{Biological functions and expression patterns of the class III PRX gene family in wheat and Ae. tauschii}

Aside from the catalytic function of reducing hydrogen peroxide to water, class III PRXs are involved in various other biological functions, including lignification, defence, development and germination [29]. Cosio et al. summarized the research methods (microarrays, transgenic plants, proteomics, and so on), expressed organs, and studied mechanisms (low oxygen response, aluminium stress, cold-inducible tolerance, etc.) of almost all 73 published $A$. thaliana PRXs [30]. In our results, we examined the expression patterns of $T$. aestivum PRXs under drought conditions and phytohormone treatments using public microarray datasets and qRT-PCR.

Some papers reported that a few PRXs were involved in root and stresses. Three plasma membrane-bound class III PRXs, namely, pmPOX1, pmPOX2b and pmPOX3, were purified in maize root and then identified by ESIMS/MS and MALDI-TOF MS [31]. The protein levels of four maize root PRXs, pmPOX1, pmPOX2a, pmPOX2b, and pmPOX3, changed in response to various stresses, including $\mathrm{H}_{2} \mathrm{O}_{2}$, SA, wounding, MeJA, Fg infection, Fusarium culmorum infection, chitosan and cantharidin [32]. In our study, we also analysed the T. aestivum PRX expression patterns in root tissue (Additional file 6: Figure S6, Additional file 20: Table S8). We noticed that some T. aestivum PRXs, such as Traes_7DL_6233C6F03.1 (I), Traes_ 1AL_38F9A30EA.1 (I) and Traes_1AL_91E56EC8C.1 (V), exhibited relatively higher expression levels in two root tissues (root in the germinating seed stage and root in the seedling stage) than in other tissues, suggesting that they might play roles in root development or metabolic processes.

Some published articles about the abiotic stress responses of PRXs are consistent with our microarray and qRT-PCR results. (1) Drought: the transcript levels of selected T. aestivum PRXs were determined under PEG6000 in two wheat cultivars ("Plainsman V", drought-tolerant; and "Cappelle Desprez", drought sensitive) [33]. The results showed that the TaPrx04 transcript was enhanced in the root apex of "Plainsman V" but decreased in "Cappelle Desprez". In our drought microarray results, we also found similar $T$. aestivum PRXs (Traes_1DS_578ADDF80.1, Traes_2AS_ 3161D54F8.1, etc.) with reverse expression trends in two wheat cultivars (drought-susceptible "WL711" and drought-tolerant "C306"). The results showed that TaPrx01, TaPrx03, TaPrx19, TaPrx68, TaPrx107 and TaPrx109-C decreased in both cultivars. In our drought microarray results, we also found similar $T$. aestivum PRXs (Traes_2AS_647C2FAA9.1, Traes_2BL_ E8A65526C.1, etc.) with downregulation in both "WL711" and "C306". (2) Hormones: we compared the four published maize PRXs [21] to our homologous $T$. aestivum PRXs by using qRT-PCR under SA treatment (Additional file 11: Figure S11). The results showed that these four homologous gene pairs exhibited similar expression trends, but their peaks of upregulation did not occur at the same time after SA treatment. 
Pathogen attack in plants leads to three defence mechanisms (cell wall lignification, production of antimicrobial metabolites, and production of ROS and RNS (reactive nitrogen species), which involve PRXs [34]. In this study, we also checked expression patterns under $F g$ infection using public microarray datasets and qRT-PCR. All eight investigated T. aestivum PRXs were upregulated in qRT-PCR and microarray results, suggesting that they might participate in the response to $F g$ infection. Additionally, our qRT-PCR results showed that four $T$. aestivum PRXs (Traes_2AL A45F6AEBE.1, Traes_5DL_011018E3C.1, Traes_1BS 871E20CF0.1 and Traes_2AS_AB001AAB7.1) were strongly upregulated (more than 100-fold) after Fg infection, suggesting that they play a central role in the FHB stress regulatory network. How do plant PRXs function in pathogen defence? A novel Marsdenia megalantha PRX was purified and shown to inhibit the phytopathogenic fungi Fusarium oxysporum and Fusarium solani through a cell membrane permeabilization mechanism [35].

In 2018, Lüthje et al. summarized the physiological functions (such as senescence, floral organ development, lignification of vessels, drought, cold and pathogen) of membrane-bound class III PRXs in Arabidopsis, barrel medic, rice and maize [36]. In this article, we selected eleven maize PRXs involved in drought or pathogen stress response and compared them with our predicted microarray results in $T$. aestivum PRXs by BLAST. Among them, only six maize PRXs had homologous probes in our microarray. However, five maize PRXs had the same physiological function as the homologous $T$. aestivum PRX (Additional file 21: Table S9). (1) Drought: T. aestivum PRXs (Traes_2BS_990895438.1 and Traes_6AS_ ADF96853B.1), which are homologous to ZmaPrx70 and ZmaPrx122 (A5H452 and A0A1D6H652), showed slight upregulation in "C306" (drought-tolerant) under drought conditions. (2) Pathogen: T. aestivum PRXs (Traes_2BS 430425C78.1 and Traes_2AL_C4CDAA081.1), which are homologous to ZmaPrx56 and ZmaPrx85 (A0A1D6IMZ0 and A0A1D6E530), showed progressive upregulation at 1-4 d after Fg infection. (3) Drought and pathogen: T. aestivum PRX (Traes_2DS_D76AB139C.1), which is homologous to ZmaPrx114 (COPPB6), showed upregulation under drought and $F g$ stresses. Our qRT-PCR results also showed that Traes_2DS_D76AB139C.1 exhibited upregulation in "Sumai-3" at $6 \mathrm{~h}$ after PEG treatment (Fig. 6).

\section{Conclusions}

In this study, we performed genome-wide identification and classification of class III PRXs in wheat, Ae. tauschii and eight other representative plants. To infer PRX evolution, the exon-intron structures of PRXs were diagrammed in these eleven plants. Some conserved exonintron structures with conserved exon phases in the PRX domain were found across species from $P$. patens to $T$. aestivum. Based on our analysis, an evolutionary model of exon-intron structures of PRX genes was proposed in which subfamily VII could be the ancient subfamily, and most subfamily V-XVIII PRXs contained the conserved exon-intron structure "001", which might origin in subfamily I. WGD, TD and syntenic analysis were performed with $T$. aestivum, $B$. distachyon and $O$. sativa PRXs. The results showed that both WGD and TD contributed to the expansion of T. aestivum PRXs. Global expression pattern analysis using public microarray datasets revealed that some PRXs could be involved in biotic and abiotic responses in wheat. qRT-PCR of selected $T$. aestivum PRXs under PEG, phytohormone and Fg treatments validated the microarray predictions. The confocal microscopy results indicating the subcellular localization of TaePRXs from different subfamilies were consistent with the website predictions. Sequencing promoters validated the predicted hormone responsive cis-elements. Our results will provide clues for researchers regarding the evolution and biological functions of PRXs.

\section{Methods \\ Identification and classification of class III PRXs in wheat, Ae. tauschii and other plants}

The genomes and proteomes of $T$. aestivum were downloaded from Ensembl Plants, release-31 (http://plants. ensembl.org/). The genomes and proteomes of ten other plants, including T. urartu, Ae. tauschii, B. distachyon, $Z$. mays, O. sativa, A. thaliana, $V$. vinifera, S. moellendorffi, $P$. patens and $C$. reinhardtii, were downloaded from Ensembl Plants, release-33. To identify the PRXs, the proteomes of all eleven plants were scanned by our local server HMMER3.1 (Pfam profile PF00141.21, peroxidase.hmm, PRX domain) and the website Pfam 30.0 (http://pfam.xfam. org/) in batch mode with an E value of 0.01. Atypical PRXs with a PRX domain covering less than $50 \%$ alignment were excluded in the following analysis. The PRX alignment of T. aestivum, T. urartu and Ae. tauschii truncated sequences in the PRX domain was performed by ClustalW v2.0 [37]. The NJ phylogenetic tree was constructed by MEGA-CC 7.0 [38] with a p-distance model and 1000 bootstrap repetitions in our local server. Similarly, the large NJ phylogenetic tree of these eleven plants was also constructed by ClustalW v2.0 and MEGA-CC 7.0. The classification of PRX subfamilies was performed by HMMER3.1, and models were generated based on the maize PRX alignments [21].

\section{Domain and exon-intron structure diagram of PRXs}

The domain and exon-intron structures of PRXs in these eleven plants were generated by our Perl and R scripts based on the corresponding GFF file information from Ensembl Plants (http://plants.ensembl.org/). The domain information was batched from Pfam 30.0 (http://pfam. xfam.org/). 


\section{Chromosome locations, duplication events and synthetic analysis of wheat PRXs}

Based on the extracted information in GFF files from Ensembl Plants (http://plants.ensembl.org/), the chromosome locations of $T$. aestivum PRXs were diagrammed using Mapchart v2.3 (https://www.wur.nl/en/show/mapchart.htm). BLASTP was performed against PRXs of T. aestivum, B. distachyon and O. sativa with an E value of e-100. Based on the GFF and BLAST results, tandem duplication and segmental duplication were searched using MCScanX [39]. The $K a$ and $K s$ values were calculated by "add_ka_ and_ks_to_collinearity.pl" in MCScanX. To search for the synthetic relations between the T. aestivum A subgenome and T. urartu, between the T. aestivum D subgenome and Ae. tauschii, BLAST was performed with an E value of e100 . Then, the $K a$ and $K s$ values were calculated by KaKs Calculator2.0 [40] with the $\gamma$-YN method.

\section{Microarray expression data analysis}

Public wheat microarray expression data was downloaded from the GEO database of NCBI. Microarray datasets of tissues and stress treatments were selected from the Affymetrix Wheat Genome Array platform GPL3802. (1) Tissues: GSE12508, thirteen tissues at defined developmental stages for wild-type wheat (cultivar Chinese Spring) [41]. (2) Abiotic stresses: cold: GSE14697, two wheat lines, freeze resistant and freeze susceptible, were compared with and without $4{ }^{\circ} \mathrm{C}$ treatment [42]; heat: GSE60351, flag leaves of the wheat cultivar "TAM 107" were sampled after $1 \mathrm{~h}$ of heat stress $\left(40{ }^{\circ} \mathrm{C}\right)$ treatment; drought: GSE87325, leaf tissues of two wheat genotypes (drought-susceptible variety "WL711" and drought-tolerant variety "C306") were collected for Affymetrix microarrays under drought stress; nutrient deficiency: GSE61679, the root tissues of wheat cultivar "Hereward" under five nutrient-deficient conditions; phytohormones: GSE103430, wheat spike tissues exposed to seven phytohormones, including IAA, GA (GA3), ABA, ET, CK (trans-zeatin), SA and MeJA. (3) biotic stresses: Fusarium head blight: GSE36283, wheat spikelets from the very susceptible spring wheat cultivar "Roblin" at 1, 2 and 4 d after Fg infection [43]; powdery mildew: GSE12936, wheat cultivar "AC Drummond" under soluble silicon ( $\mathrm{Si}$ ) and pathogen stress (B. graminis f.sp. tritici, Bgt) [44]; blast fungus: GSE31760, wheat cultivar "Renan" infected by three Magnaporthe pathogen isolates (non-adapted BR29, adapted BR32 and BR37) [45]; Hessian fly larvae: GSE34445, two wheat lines, "Molly" (containing $\mathrm{R}$ gene $\mathrm{H} 13$, resistance) and "Newton" (susceptible), were collected at 6, 12 and $24 \mathrm{~h}$ after Hessian fly egg hatching [46]; earthworms: GSE47479, wheat was inoculated with the soil-borne fungus G. graminis var. tritici (Ggt) and earthworms. Quality control and normalization of raw data were performed by RMAexpress v1.2.0 (http://www.rmaexpress.bmbolstad.com/). The mean expression levels of tissues were calculated using our Perl script. The expression levels ( $\log 2$ value of fold change, treatment vs. control) of $T$. aestivum PRXs under stress treatments were calculated by $\mathrm{R}$ software and the $\mathrm{R}$ package limma. Heat maps of $T$. aestivum PRX expression levels were generated by Mev4.9 [47].

\section{Plant material and stress treatments}

Wheat (T. aestivum L.) cultivar "Sumai-3" seeds were germinated on damp filter paper at room temperature for approximately $24 \mathrm{~h}$. The mesocotyls grew to approximately $2-3 \mathrm{~mm}$ in length, and then the seedlings were transferred into pots for growth in a greenhouse at 20$25^{\circ} \mathrm{C}$ with a photoperiod of $16 \mathrm{~h} / 8 \mathrm{~h}$. The seedlings with two leaves were used in all experiments unless stated otherwise. Then, the seedlings were subjected to drought stress (20\% (m/V) PEG-6000) for 0, 6, 12, 24, 48 and 72 $\mathrm{h}$. The seedlings were also treated with four phytohormones, $1.5 \mathrm{mM} \mathrm{SA}, 100 \mu \mathrm{M}$ MeJA, $100 \mu \mathrm{M}$ IAA and $100 \mu \mathrm{M}$ ABA, for $0,1,3,6,12,24$ and $48 \mathrm{~h}$. The spikelets of "Sumai-3" in the flowering stage were inoculated with $F g$ for 0, 12, 24, 48, 72 and 96 h, respectively. Leaves (drought and phytohormones) and spikelets $(F g)$ were separately collected and immediately frozen in liquid nitrogen and then stored at $-80^{\circ} \mathrm{C}$ for qRT-PCR. At least 30 samples of each experimental replicate were analysed with different treatments.

\section{RNA extraction and qRT-PCR}

Total RNA was isolated using the TRIzol kit (TransGen Biotech Co., Ltd., Beijing, China). The first-strand cDNA was synthesized with oligo-dT primers using TransScript First-Strand cDNA Synthesis Supermix (TransGen Biotech Co., Ltd., Beijing, China). qRT-PCR was performed in a $20-\mu$ l reaction volume using a Roche LightCycler ${ }^{\bullet}$ 480 (Roche Diagnostics GmbH, Mannheim, Germany) for three biological replicates. Wheat $\beta$-Actin was used as an internal reference. Relative mRNA levels were calculated using the $2^{-\Delta \Delta C T}$ method. The qRT-PCR primers for PEG, four phytohormones and FHB are supplied in Additional file 26: Table S14.

\section{Subcellular localization of TaePRXs}

The coding region of TaePRXs was amplified and fused to the N-terminus of eGFP, which was driven by the CaMV 35S promoter to generate pBIN35S-TaePRXeGFP. At the same time, we used a 35S:eGFP fusion construct as a control. The constructs were used for subcellular localization analysis. The positive clones were transformed into Agrobacterium EHA105. The resulting Agrobacterium culture was resuspended in an infiltration medium [10 mM 4-morpholineethanesulfonic acid 
hydrate (MES) (pH 5.6), $10 \mathrm{mM} \mathrm{MgCl}_{2}$, and $200 \mathrm{mM}$ acetosyringone) and then injected into four-week-old tobacco (Nicotiana benthamiana) leaves at an OD600 of 0.6. Transformed tobacco leaves were imaged using a confocal microscope (PERKINEIMER UITRAVIEW VOX Confocal Microscope). The TaePRXs cloning experiment was performed in leaves of the wheat cultivar "Sumai-3". Subcellular localization of the primers for TaePRXs are supplied in Additional file 27: Table S15. Predicted subcellular localization of TaePRXs was performed in WoLF PSORT (https://www.genscript.com/ wolf-psort.html?src=leftbar) and TargetP (http://www. cbs.dtu.dk/services/TargetP/).

\section{Predicted cis-acting elements and sequencing validation} For promoter analysis, $2 \mathrm{~kb}$ upstream region from the translation start codon ATG of each TaePRX was truncated by our Perl scripts, and then predicted cis-acting elements in the $(+)$ strand and $(-)$ strand of each promoter of TaePRXs were found using the PlantCARE42 database (http://bioinformatics.psb.ugent.be/webtools/ plantcare/html/). To validate these predicted cis-acting elements, total DNA was isolated, and then PCR was performed to amply 2-kb upstream promoter sequences. Gel extraction and sequencing validation were performed. The TaePRX promoter cloning experiment was performed in leaves of the wheat cultivar "Sumai-3". Primers used to clone the promoters related to predicted cis-elements are supplied in Additional file 28: Table S16.

\section{Additional files}

Additional file 1: Figure S1. Expanded phylogenetic classification of class III peroxidases using the neighbour-joining method. (a) T. aestivum, T. urartu and Ae. tauschii; (b) All investigated plants. (PDF $376 \mathrm{~kb}$ )

Additional file 2: Figure S2. Exon-intron and domain diagrams of class III peroxidases in T. aestivum, T. urartu, Ae. tauschii, B. distachyon, Z. mays, O. sativa, A. thaliana, V. vinifera, S. moellendorffii, P. patens and C. reinhardtii. The descriptions of the domain and exon phases are the same as those in Fig. 2. The lengths of the boxes and lines are scaled based on the lengths of the genes. (PDF $487 \mathrm{~kb}$ )

Additional file 3: Figure S3. Conserved exon-intron and domain diagrams of class III peroxidases in T. aestivum, B. distachyon, $S$. moellendorffii and $P$. patens. The descriptions of the domain and exon phases are the same as those in Fig. 2. The lengths of the boxes and lines are scaled based on the lengths of the genes. (PDF $30 \mathrm{~kb}$ )

Additional file 4: Figure S4. Chromosome locations of class III peroxidases in $T$. aestivum. (PDF $49 \mathrm{~kb}$ )

Additional file 5: Figure S5. Quality control of GEO microarray datasets. RLE (Relative log expression) and NUSE (Normalized unscaled standard errors) values of each GEO microarray dataset. (PDF $2638 \mathrm{~kb}$ )

Additional file 6: Figure S6. Heatmap of the expression patterns of $T$. aestivum class III peroxidase genes in different tissues. The expression patterns of 170 class III peroxidase genes in different tissues: coleoptile, root and embryo of germinating seed; root, crown and leaf of seedling; immature inflorescence; floral bracts, pistil and anthers before anthesis; 3 5 DAP (day after planting) caryopsis; 22 DAP embryos. The heatmap was generated using MeV (Multiple Experiment Viewer) software, version 4.9.
Red and green correspond to upregulation and downregulation, respectively. Normalized gene expression values and $p$ values are provided in Additional file 20: Table S8. (PDF $1350 \mathrm{~kb}$ )

Additional file 7: Figure S7. Heatmap of the expression patterns of $T$. aestivum class III peroxidase genes under abiotic stress treatments. The expression patterns of 170 class III peroxidase genes under abiotic stress treatments (cold, heat, drought and nutrient deficiency) are presented. Normalized gene expression values are provided in Additional file 21: Table S9. (PDF $1333 \mathrm{~kb}$ )

Additional file 8: Figure S8. Heatmap of the expression patterns of T. aestivum class III peroxidase genes under seven phytohormone treatments. The expression patterns of 170 class III peroxidase genes under seven phytohormone treatments, including IAA, GA (GA3), ABA, ET, CK (trans-zeatin), SA and MeJA, are presented. Normalized gene expression values are provided in Additional file 21: Table S9. (PDF $1226 \mathrm{~kb}$ )

Additional file 9: Figure S9. Upstream sequences and predicted cisacting elements related to stress and hormone responses in TaePRXs. Various predicted cis-acting elements are circled by different colours. The corresponding information is provided in Additional file 23: Table S11. (PDF $6070 \mathrm{~kb}$ )

Additional file 10: Figure S10. Sequencing data of the predicted $c$ iselements in upstream. Pair alignments were performed between upstream sequences and sequencing data. Matched cis-acting elements between them are circled by red boxes. Cis-acting elements with tiny differences are circled by green boxes. (PDF $1069 \mathrm{~kb}$ )

Additional file 11: Figure S11. qRT-PCR of four T. aestivum PRXs under four phytohormone treatments and homologous maize PRXs under SA treatment. (A) qRT-PCR of T. aestivum PRXs under SA, JA, $I A A$ and $A B A$ treatments. (B) Homologous maize PRXs under SA treatment. (PDF $912 \mathrm{~kb}$ )

Additional file 12: Figure S12. Heatmap of the expression patterns of T. aestivum class III peroxidase genes under biotic stress treatments. The expression patterns of 170 class III peroxidase genes under biotic stress treatments (Fusarium head blight, powdery mildew, blast fungus, Hessian fly larvae and earthworms) are presented. Normalized gene expression values are provided in Additional file 24: Table S12. (PDF $1418 \mathrm{~kb}$ )

Additional file 13: Table S1. Subfamily classification of class III peroxidases in the investigated plant genomes. (XLS $168 \mathrm{~kb}$ )

Additional file 14: Table S2. List of atypical class III peroxidases in the investigated plant genomes. (XLS $87 \mathrm{~kb}$ )

Additional file 15: Table S3. Chromosome locations of T. aestivum class III peroxidases. (XLS $64 \mathrm{~kb}$ )

Additional file 16: Table S4. Collinearity events and $\mathrm{Ka} / \mathrm{Ks}$ values of class III peroxidases among T. aestivum, B. distachyon and O. sativa. Sheet 1 was $\mathrm{Ka} / \mathrm{Ks}$ values of collinearity events in T. aestivum class III peroxidases; sheet 2 was $\mathrm{Ka} / \mathrm{Ks}$ values of collinearity events in all $T$. aestivum genes. Similarly, sheets 3-4 showed T. aestivum and $B$. distachyon. Sheets 5-6 showed T. aestivum and O. sativa. (XLS $5739 \mathrm{~kb}$ )

Additional file 17: Table S5. Collinearity events and $\mathrm{Ka} / \mathrm{Ks}$ values of class III peroxidases in T. aestivum subgenomes, Ae. tauschii and T. urartu. Sheet 1 was Ka/Ks values of class III peroxidase collinearity events in the $T$. aestivum A-subgenome and T. urartu. Similarly, sheet 2 showed the $T$. aestivum D-subgenome and Ae. tauschii. (XLS $58 \mathrm{~kb}$ )

Additional file 18: Table S6. Chromosome locations of tandemly arrayed T. aestivum class III peroxidases. (XLS $34 \mathrm{~kb}$ )

Additional file 19: Table S7. Public wheat expression data. (XLS 48 kb) Additional file 20: Table S8. Normalized gene expression values of 170 T. aestivum class III peroxidase genes in different tissues. (XLS 72 kb)

Additional file 21: Table S9. Normalized gene expression values of 170 T. aestivum class III peroxidase genes under abiotic stress treatments. (XLS $138 \mathrm{~kb})$

Additional file 22: Table S10. Differentially expressed $(p<0.01$ and $|F C|>1.5)$ T. aestivum class III peroxidase genes under abiotic stress treatments. (XLS $47 \mathrm{~kb})$ 
Additional file 23: Table S11. Putative cis-acting elements related to stress and hormone responses in TaePRXs promoters. (XLS $39 \mathrm{~kb}$ )

Additional file 24: Table S12. Normalized gene expression values of 170 T. aestivum class III peroxidase genes under biotic stress treatments. (XLS $111 \mathrm{~kb})$

Additional file 25: Table S13. Differentially expressed ( $p<0.01$ and $|F C|>1.5)$ T. aestivum class III peroxidase genes under biotic stress treatments. (XLS $38 \mathrm{~kb}$ )

Additional file 26: Table S14. Primers used in qRT-PCR analysis. (XLSX $40 \mathrm{~kb}$ )

Additional file 27: Table S15. Primers of TaePRXs used for subcellular localization. (XLS $20 \mathrm{~kb}$ )

Additional file 28: Table S16. Primers used to clone the promoters related to predicted cis-elements. (XLS $20 \mathrm{~kb}$ )

\section{Abbreviations}

ABA: Abscisic acid; CK: Trans-zeatin, one kind of cytokinins; DAP: Day after planting; EST: Expressed Sequence Tag; ET: Ethylene; FC: Fold change; FHB: Fusarium head blight; GA: Gibberellic acid; GEO: Gene expression omnibus; GFP: Green fluorescent protein; GST: Glutathione-S-transferase; HMM: The hidden Markov model; IAA: Indole-3-acetic acid; JA: Jasmonic acid; Ka: Non-synonymous substitution; Ks: Synonymous substitution; MeJA: Methyljasmonic acid; NJ: Neighbour-joining; NUSE: Normalized unscaled standard errors; PEG: Polyethylene glycol; PK: Protein kinase: PRX: Peroxidase; qRT-PCR: Quantitative real-time PCR; RLE: Relative log expression; RNS: Reactive nitrogen species; ROS: Reactive oxygen species; SA: Salicylic acid; SNP: Single nucleotide polymorphism; SOD: Superoxide dismutase; TaePRXs: T. aestivum PRXs; TD: Tandem duplication; WGD: Whole genome duplication

\section{Acknowledgements}

We would like to thank Dr. An-Yuan Guo (Huazhong University of Science and Technology) and Dr. Xiaoyong Sun (Shandong Agricultural University) for advice on this study.

\section{Authors' contributions}

LK and EN designed the experiment and revised the manuscript. JY performed the experiment and wrote the manuscript draft. PS and WL performed the subcellular localization and sequenced the predicted cis-elements. PS and GX performed the GRT-PCR and revised the manuscript. YZ, XM and HW revised the manuscript. All authors have read and approved the manuscript.

\section{Funding}

This work was supported by the fund of the National Natural Science Foundation of China (31520103911), the Transgenic Special Item of China (2016ZX08009-003), and the Principle Investigator of Shandong Wheat Research Team (SDAIT-01-01). This work was also supported by the funding of J.Y: A Project of Shandong Province Higher Educational Science and Technology Program (J18KA117) and the Fund for Fostering Talents of Information College (xxxy201707). The funding body had no role in the design of the study and collection, analysis, and interpretation of data and in writing the manuscript.

\section{Availability of data and materials}

The genomes, proteomes and GFF files of the investigated plants are available in Ensembl Plants (http://plants.ensembl.org/). The accession numbers of plants are T. aestivum (IWGSC1 + popseq.31), Ae. tauschii (ASM34733v1), A. thaliana (TAIR10), B. distachyon (v1.0), O. sativa (IRGSP-1.0), P. patens (ASM242v1), S. moellendorffii (v1.0), T. urartu (ASM34745v1), V. vinifera (IGGP_12x), and Z. mays (AGPv4). Public wheat microarray expression data are available in the GEO database of NCBI. The accession numbers of microarrays are GSE12508, GSE14697, GSE60351, GSE87325, GSE61679, GSE103430, GSE36283, GSE12936, GSE31760, GSE34445 and GSE47479.

\section{Ethics approval and consent to participate}

The wheat material (cultivar "Sumai-3") used in this study was obtained from the College of Agronomy, Shandong Agricultural University, Tai'an, Shandong, PR China and is publicly available for non-commercial purposes.

\section{Consent for publication}

Not applicable.

\section{Competing interests}

The authors declare that they have no competing interests.

\section{Author details}

${ }^{1}$ College of Information Science and Engineering, Shandong Agricultural University, Tai'an, Shandong 271018, People's Republic of China. ${ }^{2}$ State Key Laboratory of Crop Biology, Shandong Key Laboratory of Crop Biology, College of Agronomy, Shandong Agricultural University, Tai'an, Shandong 271018, People's Republic of China. Institute of Evolution, University of Haifa, 199 Aba Khoushy Ave., Mount Carmel, 3498838 Haifa, Israel.

Received: 27 November 2018 Accepted: 30 July 2019 Published online: 22 August 2019

\section{References}

1. Mathe C, Barre A, Jourda C, Dunand C. Evolution and expression of class III peroxidases. Arch Biochem Biophys. 2010;500(1):58-65.

2. Hiraga S, Sasaki K, Ito H, Ohashi Y, Matsui H. A large family of class III plant peroxidases. Plant Cell Physiol. 2001;42(5):462-8.

3. Hofrichter M, Ullrich R, Pecyna MJ, Liers C, Lundell T. New and classic families of secreted fungal heme peroxidases. Appl Microbiol Biotechnol. 2010:87(3):871-97.

4. Passardi F, Cosio C, Penel C, Dunand C. Peroxidases have more functions than a Swiss army knife. Plant Cell Rep. 2005;24(5):255-65.

5. Mei W, Qin Y, Song W, Li J, Zhu Y. Cotton GhPOX1 encoding plant class III peroxidase may be responsible for the high level of reactive oxygen species production that is related to cotton fiber elongation. J Genet Genomics. 2009:36(3):141-50.

6. Cosio C, Ranocha P, Francoz E, Burlat V, Zheng Y, Perry SE, Ripoll JJ, Yanofsky M, Dunand C. The class III peroxidase PRX17 is a direct target of the MADS-box transcription factor AGAMOUS-LIKE15 (AGL15) and participates in lignified tissue formation. New Phytol. 2017;213(1):250-63.

7. Raggi S, Ferrarini A, Delledonne M, Dunand C, Ranocha P, De Lorenzo G, Cervone F, Ferrari S. The Arabidopsis class III peroxidase AtPRX71 negatively regulates growth under physiological conditions and in response to cell wall damage. Plant Physiol. 2015;169(4):2513-25.

8. Herrero J, Esteban-Carrasco A, Zapata JM. Looking for Arabidopsis thaliana peroxidases involved in lignin biosynthesis. Plant Physiol Biochem. 2013;67: $77-86$.

9. Fernandez-Perez F, Vivar T, Pomar F, Pedreno MA, Novo-Uzal E. Peroxidase 4 is involved in syringyl lignin formation in Arabidopsis thaliana. J Plant Physiol. 2015;175:86-94.

10. Fernandez-Perez F, Pomar F, Pedreno MA, Novo-Uzal E. The suppression of AtPrx52 affects fibers but not xylem lignification in Arabidopsis by altering the proportion of syringyl units. Physiol Plant. 2015;154(3):395-406.

11. Herrero J, Fernandez-Perez F, Yebra T, Novo-Uzal E, Pomar F, Pedreno MA, Cuello J, Guera A, Esteban-Carrasco A, Zapata JM. Bioinformatic and functional characterization of the basic peroxidase 72 from Arabidopsis thaliana involved in lignin biosynthesis. Planta. 2013;237(6):1599-612.

12. Jia L, Xu W, Li W, Ye N, Liu R, Shi L, Bin Rahman AN, Fan M, Zhang J. Class II peroxidases are activated in proanthocyanidin-deficient Arabidopsis thaliana seeds. Ann Bot. 2013:111(5):839-47.

13. Kim BH, Kim SY, Nam KH. Genes encoding plant-specific class III peroxidases are responsible for increased cold tolerance of the brassinosteroidinsensitive 1 mutant. Mol Cells. 2012;34(6):539-48.

14. Severino FE, Brandalise M, Costa CS, Wilcken SR, Maluf MP, Goncalves W, Maia IG. CaPrx, a Coffea arabica gene encoding a putative class III peroxidase induced by root-knot nematode infection. Plant Sci. 2012;191192:35-42.

15. Wang CJ, Chan YL, Shien $\mathrm{CH}$, Yeh KW. Molecular characterization of fruitspecific class III peroxidase genes in tomato (Solanum lycopersicum). J Plant Physiol. 2015;177:83-92.

16. Kidwai M, Dhar W, Gautam N, Tiwari M, Ahmad IZ, Asif MH, Chakrabarty D. Oryza sativa class III peroxidase (OsPRX38) overexpression in Arabidopsis thaliana reduces arsenic accumulation due to apoplastic lignification. J Hazard Mater. 2018;362:383-93. 
17. Wu Y, Yang Z, How J, Xu H, Chen L, Li K. Overexpression of a peroxidase gene (AtPrx64) of Arabidopsis thaliana in tobacco improves plant's tolerance to aluminum stress. Plant Mol Biol. 2017;95(1-2):157-68.

18. Tognolli M, Penel C, Greppin H, Simon P. Analysis and expression of the class III peroxidase large gene family in Arabidopsis thaliana. Gene. 2002; 288(1-2):129-38.

19. Passardi F, Longet D, Penel C, Dunand C. The class III peroxidase multigenic family in rice and its evolution in land plants. Phytochemistry. 2004;65(13): 1879-93.

20. Ren LL, Liu YJ, Liu HJ, Qian TT, Qi LW, Wang XR, Zeng QY. Subcellular relocalization and positive selection play key roles in the retention of duplicate genes of Populus class III peroxidase family. Plant Cell. 2014;26(6): 2404-19.

21. Wang Y, Wang Q, Zhao Y, Han G, Zhu S. Systematic analysis of maize class III peroxidase gene family reveals a conserved subfamily involved in abiotic stress response. Gene. 2015;566(1):95-108.

22. Cao Y, Han Y, Meng D, Li D, Jin Q, Lin Y, Cai Y. Structural, evolutionary, and functional analysis of the class III peroxidase gene family in Chinese pear (Pyrus bretschneideri). Front Plant Sci. 2016;7:1874.

23. Bakalovic N, Passardi F, loannidis V, Cosio C, Penel C, Falquet L, Dunand C. PeroxiBase: a class III plant peroxidase database. Phytochemistry. 2006:67(6):534-9.

24. Lazzarotto F, Turchetto-Zolet AC, Margis-Pinheiro M. Revisiting the nonanimal peroxidase superfamily. Trends Plant Sci. 2015:20(12):807-13.

25. Kupriyanova EV, Mamoshina PO, Ezhova TA. Evolutionary divergence of Arabidopsis thaliana classical peroxidases. Biochemistry (Mosc). 2015;80(10): 1362-72.

26. Marcussen T, Sandve SR, Heier L, Spannagl M, Pfeifer M, Jakobsen KS, Wulff BB, Steuernagel B, Mayer KF, Olsen OA. Ancient hybridizations among the ancestral genomes of bread wheat. Science. 2014;345(6194):1250092.

27. Yan J, Su P, Wei Z, Nevo E, Kong L. Genome-wide identification, classification, evolutionary analysis and gene expression patterns of the protein kinase gene family in wheat and Aegilops tauschii. Plant Mol Biol. 2017:95(3):227-42.

28. Catalan P, Kellogg EA, Olmstead RG. Phylogeny of Poaceae subfamily Pooideae based on chloroplast ndhF gene sequences. Mol Phylogenet Evol. 1997:8(2):150-66.

29. Shigeto J, Tsutsumi Y. Diverse functions and reactions of class III peroxidases. New Phytol. 2016;209(4):1395-402.

30. Cosio C, Dunand C. Specific functions of individual class III peroxidase genes. J Exp Bot. 2009;60(2):391-408.

31. Mika A, Buck F, Luthje S. Membrane-bound class III peroxidases: identification, biochemical properties and sequence analysis of isoenzymes purified from maize (Zea mays L.) roots. J Proteome. 2008;71(4):412-24.

32. Mika A, Boenisch MJ, Hopff D, Luthje S. Membrane-bound guaiacol peroxidases from maize (Zea mays L.) roots are regulated by methyl jasmonate, salicylic acid, and pathogen elicitors. J Exp Bot. 2010;61(3):831-41.

33. Csiszar J, Galle A, Horvath E, Dancso P, Gombos M, Vary Z, Erdei L, Gyorgyey J, Tari I. Different peroxidase activities and expression of abiotic stressrelated peroxidases in apical root segments of wheat genotypes with different drought stress tolerance under osmotic stress. Plant Physiol Biochem. 2012;52:119-29.

34. Almagro L, Gomez Ros LV, Belchi-Navarro S, Bru R, Ros Barcelo A, Pedreno MA. Class III peroxidases in plant defence reactions. J Exp Bot. 2009;60(2):377-90.

35. Oliveira HP, Silva RGG, Oliveira JTA, Sousa DOB, Pereira ML, Souza PFN, Soares AA, Gomes VM, Monteiro-Moreira ACO, Moreno F, et al. A novel peroxidase purified from Marsdenia megalantha latex inhibits phytopathogenic fungi mediated by cell membrane permeabilization. Int J Biol Macromol. 2017;96:743-53.

36. Luthje S, Martinez-Cortes T. Membrane-Bound Class III Peroxidases: Unexpected enzymes with exciting functions. Int J Mol Sci. 2018;19:10.

37. Larkin MA, Blackshields G, Brown NP, Chenna R, McGettigan PA, McWilliam H, Valentin F, Wallace IM, Wilm A, Lopez R, et al. Clustal W and Clustal X version 2.0. Bioinformatics. 2007;23(21):2947-8.

38. Kumar S, Stecher G, Tamura K. MEGA7: molecular evolutionary genetics analysis version 7.0 for bigger datasets. Mol Biol Evol. 2016;33(7):1870-4.

39. Wang Y, Tang H, Debarry JD, Tan X, Li J, Wang X, Lee TH, Jin H, Marler B, Guo H, et al. MCScanX: a toolkit for detection and evolutionary analysis of gene synteny and collinearity. Nucleic Acids Res. 2012;40(7):e49.

40. Wang D, Zhang Y, Zhang Z, Zhu J, Yu J. KaKs_Calculator 2.0: a toolkit incorporating gamma-series methods and sliding window strategies. Genomics Proteomics Bioinformatics. 2010;8(1):77-80.
41. Schreiber AW, Sutton T, Caldo RA, Kalashyan E, Lovell B, Mayo G, Muehlbauer GJ, Druka A, Waugh R, Wise RP, et al. Comparative transcriptomics in the Triticeae. BMC Genomics. 2009;10:285.

42. Sutton F, Chen DG, Ge X, Kenefick D. Cbf genes of the Fr-A2 allele are differentially regulated between long-term cold acclimated crown tissue of freeze-resistant and - susceptible, winter wheat mutant lines. BMC Plant Biol. 2009;9:34.

43. Wojcik PI, Ouellet T, Balcerzak M, Dzwinel W. Identification of biomarker genes for resistance to a pathogen by a novel method for meta-analysis of single-channel microarray datasets. J Bioinforma Comput Biol. 2015;13(4): 1550013.

44. Chain F, Cote-Beaulieu C, Belzile F, Menzies JG, Belanger RR. A comprehensive transcriptomic analysis of the effect of silicon on wheat plants under control and pathogen stress conditions. Mol Plant-Microbe Interact. 2009;22(11):1323-30.

45. Tufan HA, McGrann GR, Magusin A, Morel JB, Miche L, Boyd LA. Wheat blast: histopathology and transcriptome reprogramming in response to adapted and nonadapted Magnaporthe isolates. New Phytol. 2009;184(2):473-84.

46. Zhu L, Liu X, Wang H, Khajuria C, Reese JC, Whitworth RJ, Welti R, Chen MS. Rapid mobilization of membrane lipids in wheat leaf sheaths during incompatible interactions with hessian fly. Mol Plant-Microbe Interact. 2012; 25(7):920-30.

47. Saeed Al, Sharov V, White J, Li J, Liang W, Bhagabati N, Braisted J, Klapa M, Currier T, Thiagarajan M, et al. TM4: a free, open-source system for microarray data management and analysis. Biotechniques. 2003;34(2):374-8.

\section{Publisher's Note}

Springer Nature remains neutral with regard to jurisdictional claims in published maps and institutional affiliations.

\section{Ready to submit your research? Choose BMC and benefit from:}

- fast, convenient online submission

- thorough peer review by experienced researchers in your field

- rapid publication on acceptance

- support for research data, including large and complex data types

- gold Open Access which fosters wider collaboration and increased citations

- maximum visibility for your research: over $100 \mathrm{M}$ website views per year

At $\mathrm{BMC}$, research is always in progress.

Learn more biomedcentral.com/submissions 